\title{
Synthesis and structural analysis of anilides of
} glucuronic acid and orientation of groups on carbohydrate scaffolding.

Manuela Tosin, Colin O'Brien, Geraldine M. Fitzpatrick, Helge Müller-Bunz, W. Kenneth Glass and Paul V. Murphy*

Centre for Synthesis and Chemical Biology, Department of Chemistry, Conway Institute of Biomolecular and Biomedical Research, University College Dublin, Belfield, Dublin 4, Ireland

Paul.V.Murphy@ucd.ie

\section{Index to Supporting Information}

Experimental Procedures: $\quad$ S2-S14

Selected IR Spectra: $\quad$ S15-S18

${ }^{1}$ H-NMR Spectra ${ }^{1} \quad$ S19-S43

Selected additional NMR Spectra S44-S46

\footnotetext{
${ }^{1}$ The NMR spectra for $6,7,8,9,10,20,21,22,23,25 \mathrm{~g}, 31$ and 32 are available in the supporting information section that accompanies the following publication: Tosin, M.; Murphy, P. V.; Org. Lett. 2002; 4 (21); 3675-3678. DOI: $10.1021 / \mathrm{ol} 026629 \mathrm{j}$
} 


\section{Experimental}

\section{General}

Optical rotations were determined with at the sodium D line at $20{ }^{\circ} \mathrm{C} .{ }^{1} \mathrm{H}(300$ and $500 \mathrm{Mz})$ and ${ }^{13} \mathrm{C}$ NMR spectra (75 MHz or $125 \mathrm{MHz}$ ) were recorded with a pulsed field gradient accessory. All ${ }^{13} \mathrm{C}$ spectra given are proton-decoupled. Chemical shifts are reported relative to internal $\mathrm{Me}_{4} \mathrm{Si}$ in $\mathrm{CDCl}_{3}(\delta 0.0)$ or $\mathrm{HOD}$ for $\mathrm{D}_{2} \mathrm{O}(\delta 4.79)$ for ${ }^{1} \mathrm{H}$ and $(\delta 77.16)$ for ${ }^{13} \mathrm{C} .{ }^{13} \mathrm{C}$ signals were assigned with the aid of DEPT-135. ${ }^{1} \mathrm{H}$ signals were assigned with the aid of 2D-COSY. Homonuclear coupling constants $(\mathrm{Hz})$ were determined from the corresponding ${ }^{1} \mathrm{H}$ spectra. The use of presaturation for solvent suppression was carried out in recording the ${ }^{1} \mathrm{H}-\mathrm{NMR}$ spectrum of 16. TLC was performed on aluminium sheets precoated with Silica Gel $60\left(\mathrm{HF}_{254}\right.$, E. Merck) and spots visualized by $\mathrm{UV}$ and charring with $\mathrm{H}_{2} \mathrm{SO}_{4}-\mathrm{EtOH}$ (1:20). Flash Column Chromatography was carried out with Silica Gel 60 (0.040-0.630 mm, E. Merck) and using a stepwise solvent polarity gradient (starting from the conditions indicated in each case and increasing the polarity) correlated with TLC mobility. Chromatography solvents used were EtOAc (Riedel-deHaen), $\mathrm{MeOH}$ (Riedel-deHaen) and petroleum ether (b.p. $40-60^{\circ} \mathrm{C}, \mathrm{BDH}$ laboratory supplies). Reaction solvents were freshly dried and distilled where stated: Acetonitrile, toluene and $\mathrm{CH}_{2} \mathrm{Cl}_{2}$ from calcium hydride, methanol from magnesium turnings and THF from sodium/benzophenone; anhydrous DMF and pyridine were used as purchased from SigmaAldrich. For preparative HPLC acetonitrile/water mixtures were used as eluant with a flow rate of $10 \mathrm{~mL} / \mathrm{min}$. The semi preparative columns used were YMC-Pack C-4 (S-10 $\mu \mathrm{m}, 250$ x 20mm)

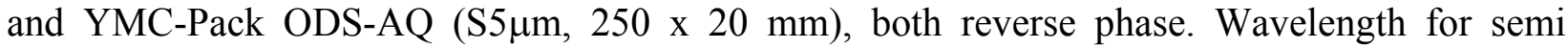
preparative HPLC was $220 \mathrm{~nm}$. 


\section{NOE and ROE Spectroscopy Experiments}

These NMR spectra were collected on the $500 \mathrm{MHz}$ Spectrometer. All samples were made up in $\mathrm{CDCl}_{3}$ or $\mathrm{D}_{2} \mathrm{O}$ or $\mathrm{D}_{2} \mathrm{O}: \mathrm{H}_{2} \mathrm{O}(10: 90)$ at concentrations of $2.0-10.0 \mathrm{mg} / \mathrm{mL}$. The spectra were run at temperatures of $5{ }^{\circ} \mathrm{C}$ or $30^{\circ} \mathrm{C}$ with water suppression (transmitter presat ) where appropriate. 2D NOESY $\left(\tau_{\text {mix }}=0.4-1.0 \mathrm{~s}\right)$ and 2D TROESY (transverse-ROESY) spectra $\left(\tau_{\operatorname{mix}}=0.4-0.5 \mathrm{~s}\right)$ the phase-sensitive mode using States-TPPI for F1-quadrature detection in the indirect dimension with relaxation delays of 2.5 seconds (2048 in F2 and 1024 in F1 points were used). In the TROESY sequence the strength of the pulsed spin lock field(B1) was 2.2KHz. The 1D-NOE experiments were collected using a double pulsed field gradient spin echo 1D NOE sequence (DPFGNOE).

\section{IR Spectroscopic studies}

The data in Table 4 was obtained on an instrument with resolution of $2 \mathrm{~cm}^{-1}$ and spectra were recorded from 5000 to $400 \mathrm{~cm}^{-1}$. The solution studies in $\mathrm{CDCl}_{3}$ and $\mathrm{CHCl}_{3}$ were recorded in 1 $\mathrm{mm} \mathrm{KBr}$ cells at concentrations of compounds of $1-2 \mathrm{mg} / \mathrm{mL}$ and for measurements in $\mathrm{CCl}_{4}$ were recorded at concentrations $<0.5 \mathrm{mg} / \mathrm{mL}$. The absorbtion frequencies obtained for secondary amides were checked for 9 over a range of concentrations $(0.6-21 \mathrm{mg} / \mathrm{mL}$, see below) and no variation was observed. Spectra recorded in the solid state were of pure solids and measured by diffuse reflectance

Preparation of Anhydride 6. D-Glucuronic acid (5.0 g, $25.24 \mathrm{mmol})$ was suspended in acetic anhydride $(75 \mathrm{~mL})$ and stirred at $\mathrm{O}{ }^{\circ} \mathrm{C}$. Iodine $(350 \mathrm{mg}, 1.65 \mathrm{mmol})$ was added slowly and the red solution was stirred for $2 \mathrm{~h}$ on ice and for further $3 \mathrm{~h}$ at room temperature. Acetic anhydride was mostly removed in vacuo and the remaining mixture taken up in dichloromethane 
$(70 \mathrm{~mL})$. The organic layer was then washed twice with $\mathrm{Na}_{2} \mathrm{~S}_{2} \mathrm{O}_{3}(1.0 \mathrm{M}, 80 \mathrm{~mL})$, dried, $\left(\mathrm{MgSO}_{4}\right)$ filtered and concentrated to afford 4 as a white solid $(10.12 \mathrm{~g}, 99 \%)$. The $\beta$-anomer can be separated from its $\alpha$-anomer (present as minor impurity) by recrystallization from dichloromethane/petroleum ether (70-80\% yield); ${ }^{1} \mathrm{H}-\mathrm{NMR}\left(300 \mathrm{MHz}, \mathrm{CDCl}_{3}\right): \delta 5.82(\mathrm{~d}, 1 \mathrm{H}$, $\left.J_{1,2} 6.9, \mathrm{H}-1\right), 5.38$ (apt t, $\left.1 \mathrm{H}, J=9.0, \mathrm{H}-4\right), 5.29($ apt t $, 1 \mathrm{H}, J$ 8.5, H-3), 5.12 (dd, $1 \mathrm{H}, \mathrm{H}-2), 4.34$ (d, $1 \mathrm{H}, \mathrm{H}-5), 2.28\left(\mathrm{~s}, 3 \mathrm{H}, \mathrm{COOCOCH}_{3}\right), 2.13,2.06,2.05,2.04$ (each s, each $3 \mathrm{H}$, each $\mathrm{COCH}_{3}$ );

${ }^{13} \mathrm{C}-\mathrm{NMR}\left(\mathrm{CDCl}_{3}\right): \delta 170.0,169.5,169.4,168.8165 .0$ (each s, each $\left.\mathrm{C}=\mathrm{O}\right), 91.6(\mathrm{~d}, \mathrm{C}-1), 73.3$, 71.6, 70.4, 68.3 (each d, C-2-5), 22.2 (q, $\mathrm{COOCOCH}_{3}$ ), 20.9, 20.4 (each q, each $\mathrm{COCH}_{3}$ ), 20.7 (2 q, overlapping, each $\mathrm{COCH}_{3}$ ); CI-HRMS: Found 422.1300, required $422.1299\left[\mathrm{M}+\mathrm{NH}_{4}\right]^{+}$.

\section{Preparation of 1,2,3,4-Tetra-O-acetyl- $\beta$-D-glucopyranuronic acid 7: The anhydride 6 (9.61}

g, $23.77 \mathrm{mmol})$ was dissolved in water and THF $(180 \mathrm{~mL}, 1: 2)$ and stirred overnight. The solution was concentrated and the product extracted into dichloromethane, dried on $\mathrm{MgSO}_{4}$ and filtered. After evaporation in vacuo a white fluffy material was obtained and identified as 7 (7.81 g, 91\%). Compound 7 can also be obtained more quickly stirring 6 in water/THF for 3 hours and evaporating the solvents directly from the reaction mixture without any workup (97-99\% yield); ${ }^{1} \mathrm{H}-\mathrm{NMR}\left(300 \mathrm{MHz}, \mathrm{CDCl}_{3}\right): \delta 5.80\left(\mathrm{~d}, 1 \mathrm{H}, J_{1,2} 7.5, \mathrm{H}-1\right), 5.31(\mathrm{~m}, 2 \mathrm{H}, \mathrm{H}-3$ and $\mathrm{H}-4$ overlapping), 5.14 (m, 1H, $\left.J_{2,3} 9.0, \mathrm{H}-2\right), 4.24$ (m, 1H, $\left.J_{4,5} 9.5, \mathrm{H}-5\right), 3.46$ (br s, $1 \mathrm{H}, \mathrm{COOH}$ ), 2.12, 2.05, 2.04, 2.03 (each s, each $3 \mathrm{H}$, each $\left.\mathrm{COCH}_{3}\right) ;{ }^{13} \mathrm{C}-\mathrm{NMR}\left(\mathrm{CDCl}_{3}\right): \delta 170.2(\mathrm{~s}, \mathrm{COOH})$, 169.9, 169.5, 169.1 (each s, each $\mathrm{COCH}_{3}$ ), 91.5 (d, C-1), 72.7, 72.1, 70.4, 68.9 (each d, C-2-C5), 20.9 (q, $\mathrm{COCH}_{3}$ ), 20.7 (3 q, overlapping, each $\mathrm{COCH}_{3}$ ); CI-LRMS: $380\left[\mathrm{M}+\mathrm{NH}_{4}\right]^{+}, 320$ $\left[\mathrm{M}+\mathrm{H}-\mathrm{CH}_{3} \mathrm{CO}\right]^{+}, 303[\mathrm{M}-\mathrm{OAc}]^{+}, 243[\mathrm{M}+\mathrm{H}-2 \mathrm{HOAc}]^{+}$. 
Synthesis and structural analysis of anilides of glucuronic acid......Tosin, O'Brien et al. S5

Preparation of amides 8 and 9 from anhydride 6: $\quad$ To a solution of 6 in dry dichloromethane $(0.1 \mathrm{~g} / \mathrm{mL})$ and under $\mathrm{N}_{2}$ atmosphere an equivalent of primary amine is added, and the reaction mixture is stirred overnight. The product is extracted into dichloromethane and washed with $\mathrm{HCl}$ (dil.), $\mathrm{NaHCO}_{3}$ (satd.) and deionised water and dried $\left(\mathrm{MgSO}_{4}\right)$. The organic layer was concentrated after filtration to afford the amide as a white solid which can be further purified by recrystallization from $\mathrm{Et}_{2} \mathrm{O}$ (or dichloromethane/petroleum ether) or by column chromatography (EtOAc/pet.ether gradient).

Analytical data for 8: $\quad{ }^{1} \mathrm{H}-\mathrm{NMR}\left(300 \mathrm{MHz}, \mathrm{CDCl}_{3}\right): \delta 6.10\left(\mathrm{br} \mathrm{d}, 1 \mathrm{H}, J_{\mathrm{C} H-\mathrm{N} H} 7.8, \mathrm{~N} H\right)$, 5.75 (d, 1H, $J_{1,2} 8.0, \mathrm{H}-1$ ), 5.30 (apt t, 1H, $J_{2,3} 9.0, J_{3,4} 9.3, \mathrm{H}-3$ ), 5.18 (apt t, 1H, $J_{4,5} 9.7, \mathrm{H}-4$ ), $5.11(\mathrm{dd}, 1 \mathrm{H}, \mathrm{H}-2), 4.02$ (d, 1H, H-5), $3.96\left(\mathrm{~m}, 1 \mathrm{H}, \mathrm{CH}\left(\mathrm{CH}_{3}\right)_{2}\right), 2.14,2.07,2.02$ (each s, each $3 \mathrm{H}$, each $\left.\mathrm{COCH}_{3}\right), 1.15\left(\mathrm{~d}, 6 \mathrm{H}, J\right.$ 6.6, $\left.\mathrm{CH}\left(\mathrm{CH}_{3}\right)_{2}\right) ;{ }^{13} \mathrm{C}-\mathrm{NMR}\left(\mathrm{CDCl}_{3}\right): \delta 170.0,169.8,169.5$, 169.0 (each s, each $\mathrm{COCH}_{3}$ ), 165.3 (s, $\left.\mathrm{CONH}\right), 91.6$ (d, C-1), 73.4, 72.3, 70.6, 69.3 (each d, C2- C-5), $41.6\left(\mathrm{~d}, \mathrm{CH}\left(\mathrm{CH}_{3}\right)_{2}\right), 22.7,22.6$ (each q, each $\left.\mathrm{CH}\left(\mathrm{CH}_{3}\right)_{2}\right), 21.0,20.9$ (each q, each $\mathrm{COCH}_{3}$ ), 20.8 (2 q, overlapping, each $\mathrm{COCH}_{3}$ ); CI-HRMS: Found 404.1556, required 404.1557 $[\mathrm{M}+\mathrm{H}]^{+}$.

Analytical data for 9: $\quad{ }^{1} \mathrm{H}-\mathrm{NMR}\left(300 \mathrm{MHz}, \mathrm{CDCl}_{3}\right): \delta 7.94(\mathrm{~s}, 1 \mathrm{H}, \mathrm{NH}), 7.47(\mathrm{dd}, 2 \mathrm{H}, J$ 8.2, J1.1, Ar H), 7.32 (apt t, J 7.5, 2H, Ar H), 7.13 (apt t, 1H, Ar H), 5.85 (d, 1H, $J_{1,2} 7.3, \mathrm{H}-1$ ), $5.36\left(\mathrm{~m}, 2 \mathrm{H}, \mathrm{H}-3\right.$ and H-4), $5.16\left(\mathrm{~m}, 1 \mathrm{H}, J_{2,3} 8.9, \mathrm{H}-2\right), 4.24\left(\mathrm{~m}, 1 \mathrm{H}, J_{4,5} 9.3, \mathrm{H}-5\right), 2.14,2.10$, 2.07, 2.03 (each s, each 3H, each $\left.\mathrm{COCH}_{3}\right) ;{ }^{13} \mathrm{C}-\mathrm{NMR}\left(\mathrm{CDCl}_{3}\right): \delta 170.0,169.8,169.5,169.1$ (each s, each $\mathrm{COCH}_{3}$ ), 164.3 (s, CONH), 136.7 (s, Ar C ipso), 129.3 (d, Ar C-b and C-b'), 125.3 (d, Ar C-c), 120.7 (d, Ar C-a and C-a'), 91.9 (d, C-1), 73.7, 72.1, 70.8, 69.9 (each d, C-2-C-5), 21.0, 
20.9 (each q, each $\mathrm{COCH}_{3}$ ), 20.8 (2 q, overlapping, each $\mathrm{COCH}_{3}$ ); CI-HRMS: Found 455.1663, required $455.1666\left[\mathrm{M}+\mathrm{NH}_{4}\right]^{+}$.

Preparation of amides from 7. Dicyclohexylcarbodiimide (1 eq) was added to a solution of 7 in dry dichloromethane $(0.1 \mathrm{~g} / \mathrm{mL})$ at $0{ }^{\circ} \mathrm{C}$ and under $\mathrm{N}_{2}$. Formation of a white precipitate was observed and after 10 minutes the amine was added and after a further 60 minutes the reaction was judged complete as there was no starting material $3\left(\mathrm{R}_{\mathrm{F}}=0.0\right.$, EtOAc / petroleum ether 1:1) detected by TLC. The solution was diluted with dichloromethane, filtered and washed with $\mathrm{NaHCO}_{3}$ (satd. aq.) and $\mathrm{HCl}(1 \mathrm{M})$. The organic layer was dried $\left(\mathrm{MgSO}_{4}\right)$ and solvent removed. This gave the amide product as a white solid. This was recrystallized from dichloromethane/petroleum ether or purified by column chromatography (EtOAc/pet.ether gradient).

\section{Analytical data for 1,2,3,4-Tetra- $O$-acetyl- $\beta$-D-glucopyranuron- $N$-methyl- $N$-phenylamide}

10. ${ }^{1} \mathrm{H}-\mathrm{NMR}\left(300 \mathrm{MHz}, \mathrm{CDCl}_{3}\right): \delta 7.49$ (apt t, $\left.2 \mathrm{H}, J 8.0, \mathrm{Ar} \mathrm{H}\right), 7.41$ (apt t, 1H, Ar H), 7.24 (d, 2H, Ar H), 5.57 (apt t, 1H, J9.4, H-4), 5.31 (d, 1H, $J_{1,2} 8.0, \mathrm{H}-1$ ), 5.18 (apt t, 1H, $J$ 9.4, H-2), 5.03 (apt t, 1H, J 9.4, H-3), 4.04 (d, 1H, $J_{4,5} 9.8, \mathrm{H}-5$ ), 3.30 (s, 3H, OCH $H_{3}$ ), 2.11 (s, 3H, $\mathrm{COCH}_{3}$ ), 1.98 (s, 6H, $2 \mathrm{COCH}_{3}$, overlapping), $1.97\left(\mathrm{~s}, 3 \mathrm{H}, \mathrm{COCH}_{3}\right) ;{ }^{13} \mathrm{C}-\mathrm{NMR}\left(\mathrm{CDCl}_{3}\right): \delta$ 170.6, 169.1, 169.0, 168.8 (each s, each $\mathrm{COCH}_{3}$ ), 164.8 (s, $C \mathrm{ONMePh}$ ), 142.4 (s, aromatic C), 130.3, 128.6, 127.1 (each d, aromatic CH), 91.8 (d, C-1), 73.0, 70.5, 70.1, 69.6 (each d, C-2-5), 37.9 (q, N $\mathrm{CH}_{3} \mathrm{Ph}$ ), 21.0 (q, $\mathrm{COCH}_{3}$ ), 20.8 (two q, overlapping, each $\mathrm{COCH}_{3}$ ), 20.7 (q, each $\mathrm{COCH}_{3}$ ); CIHRMS: Found 474.1381, required $474.1376[\mathrm{M}+\mathrm{Na}]^{+}$. 
2,3,4-Tri-O-acetyl- $\beta$-D-glucopyranurono-6,1-lactone 22. Anhydride 6 (1.0 g, $2.5 \mathrm{mmol})$ was dissolved in dry dichloromethane $(20 \mathrm{ml})$ under $\mathrm{N}_{2}$ atmosphere and $\mathrm{SnCl}_{4}(0.9 \mathrm{~mL}, 3 \mathrm{eq})$ was added. The reaction mixture was left stirring overnight. Then the solution was diluted with dichloromethane $(20 \mathrm{~mL})$ and satd. $\mathrm{NaHCO}_{3}(50 \mathrm{~mL})$ was added. It was stirred for $30 \mathrm{~min}$. and then filtered using Celite. Organic layer was washed with satd. $\mathrm{NaHCO}_{3}(2 \times 50 \mathrm{~mL})$, dried $\left(\mathrm{Na}_{2} \mathrm{SO}_{4}\right)$ and concentrated. Recrystallization from ethanol provide the pure lactone $22(0.67 \mathrm{~g}$, $90 \%$ ) as white crystalline product. Analytical data was in agreement with that previously reported; ${ }^{1} \mathrm{H}-\mathrm{NMR}\left(300 \mathrm{MHz}, \mathrm{CDCl}_{3}\right): \delta 5.88(\mathrm{~m}, 1 \mathrm{H}, \mathrm{H}-1), 4.89(\mathrm{~m}, 1 \mathrm{H}, \mathrm{H}-3), 4.76(\mathrm{~m}, 1 \mathrm{H}, \mathrm{H}-$ 4), $4.72\left(\mathrm{dd}, 1 \mathrm{H}, J_{1,2} 1.5, J_{2,3} 1.5, \mathrm{H}-2\right), 4.55$ (apt t, $\left.1 \mathrm{H}, J_{3,4} 2.0, J_{4,5} 2.0, \mathrm{H}-5\right), 2.12$ (s, 6H, 2 $\mathrm{COCH}_{3}$, overlapping), $2.04\left(\mathrm{~s}, 3 \mathrm{H}, \mathrm{COCH}_{3}\right) ;{ }^{13} \mathrm{C}-\mathrm{NMR}\left(\mathrm{CDCl}_{3}\right): \delta 169.6,169.5,168.7$ (each s, each $\mathrm{COCH}_{3}$ ), 168.0 (s, COO), 100.6 (d, C-1), 71.3, 69.2, 66.3, 66.1 (each d, C-2 - 5), 20.9 (q, $\mathrm{COCH}_{3}$ ), 20.8 (2 q, overlapping, each $\mathrm{COCH}_{3}$ ); CI-HRMS: Found 320.0984, required 320.0982 $\left[\mathrm{M}+\mathrm{NH}_{4}\right]^{+}$.

Preparation of 17 and 23. The lactone $22(1 \mathrm{eq})$ was dissolved in dry $\mathrm{CH}_{2} \mathrm{Cl}_{2}(5 \mathrm{~mL})$ under $\mathrm{N}_{2}$ atmosphere and $\mathrm{SnCl}_{4}$ (Aldrich, $0.5 \mathrm{eq}$ ) and azidotrimethylsilane (2.5 eq) were added and the reaction mixture was left to stir for $16 \mathrm{~h}$. The mixture was then diluted with dichloromethane and satd. $\mathrm{NaHCO}_{3}$ was added and this was stirred for $30 \mathrm{~min}$. The mixture was filtered using Celite and the layers then separated and the aqueous layer was acidified with acetic acid. The product was extracted with EtOAc (up to $10 \times 10 \mathrm{~mL}$ washes), dried $\left(\mathrm{Na}_{2} \mathrm{SO}_{4}\right)$, filtered and the solvent was removed to give the 22:78 mixture of 17 and $23(84 \%)$. Analytical data for 23: ${ }^{1} \mathrm{H}-$ NMR (300 MHz, $\left.\mathrm{D}_{2} \mathrm{O}\right): \delta 5.85\left(\mathrm{~d}, 1 \mathrm{H}, J_{1,2} 4.0, \mathrm{H}-1\right), 5.37$ (t, $\left.1 \mathrm{H}, J 9.2, \mathrm{H}-3\right), 5.24(\mathrm{t}, 1 \mathrm{H}, J 9.2, \mathrm{H}-$ 4), 5.15 (dd, 1H, H-2), 4.67 (d, 1H, H-5), 2.13, 2.11, 2.08 (each s, each 3H, each $\left.\mathrm{COCH}_{3}\right) ;{ }^{13} \mathrm{C}-$ 
NMR ( $\left.\mathrm{D}_{2} \mathrm{O}\right): \delta 173.7(\mathrm{~s}, \mathrm{COOH}), 173.3,172.7,172.6$ (each s, each $\left.\mathrm{COCH}_{3}\right), 85.6(\mathrm{~d}, \mathrm{C}-1), 71.7$, 70.1, 69.8, 69.7 (each d, C-2-5), 20.4, 20.3, 20.2 (each q, each $\mathrm{COCH}_{3}$ ); CI-HRMS: Found 368.0702, required $368.0706[\mathrm{M}+\mathrm{Na}]^{+}$.

\section{Preparation of secondary amides.}

The mixture of $\mathbf{1 7}$ and $\mathbf{2 3}$, prepared from lactone $\mathbf{2 2}$ or a sample containing only $\mathbf{1 7}$ was dissolved in dichloromethane $(0.04 \mathrm{mmol} / \mathrm{mL})$ under $\mathrm{N}_{2}$ and cooled to $0{ }^{\circ} \mathrm{C}$. Oxalyl chloride $(1.2$ eq) and anhydrous DMF $(2.5 \mathrm{mmol} / \mathrm{ml})$ were added and the resulting mixture stirred for $30 \mathrm{~min}$ at

$0{ }^{\circ} \mathrm{C}$ and for a further $30 \mathrm{~min}$ at room temperature. In a separate vessel the amine (1.2 eq) was stirred in anhydrous dichloromethane in the presence of pyridine ( 2 eq) and $4 \AA$ molecular sieves until complete dissolution had occurred. The reaction mixture containing the acid chloride was cooled to $0{ }^{\circ} \mathrm{C}$ and the solution containing the amine then added dropwise. Stirring was continued at $0{ }^{\circ} \mathrm{C}$ for $30 \mathrm{~min}$. and then at room temperature for a further $3 \mathrm{~h}$. Dichloromethane was added and an equal volume of satd. $\mathrm{NaHCO}_{3}$ and stirring continued for $30 \mathrm{~min}$. The layers were separated and the organic layer washed with satd. $\mathrm{NaHCO}_{3}$ (x 2), $1.0 \mathrm{M} \mathrm{HCl}$ (x 2) and brine. The organic layer was dried $\left(\mathrm{MgSO}_{4}\right)$, filtered and the solvent removed. The amides were obtained as an inseparable mixture of anomers after column chromatography using EtOAc and cyclohexane (1:2) as eluant.

Preparation of N-methylated tertiary amides. The secondary amide was dissolved in anhydrous DMF $(0.02 \mathrm{~g} / \mathrm{mL})$ under an atmosphere of $\mathrm{N}_{2}$. Sodium hydride $(60 \%$ dispersion in mineral oil) ( 3 eq.) was then added and the reaction mixture cooled to $0^{\circ} \mathrm{C}$. Iodomethane ( 3 eq.) was added and the mixture stirred for an hour. The reaction was monitored by TLC (EtOAc/ 
cyclohexane 1:1). Upon completion EtOAc and water were added and stirred for 20-30 min. Layers were separated and the organic layer was washed with water, dried $\left(\mathrm{MgSO}_{4}\right)$ and concentrated. Compounds were purified further by column chromatography using a EtOAc and cyclohexane mixture (1:3).

\section{Analytical data for protected tertiary amides:}

\section{2,3,4-Tri-O-acetyl-1-azido-1-deoxy- $\alpha / \beta$-D-glucopyranuronic}

acid,

methylphenylamide. ${ }^{1} \mathrm{H}$ NMR data for $\alpha$-anomer: $\left(300 \mathrm{MHz}, \mathrm{CDCl}_{3}\right): \delta 7.28(\mathrm{~d}, 2 \mathrm{H}, J 8.0, \mathrm{Ar}$ H), $7.12\left(\mathrm{~d}, 2 \mathrm{H}, J\right.$ 8.1, Ar H), $5.51\left(\mathrm{~d}, 1 \mathrm{H}, J_{1,2} 4.1, \mathrm{H}-1\right), 5.48\left(\mathrm{t}, 1 \mathrm{H}, J_{3,4} 9.6, J_{4,5}\right.$ 9.9, H-4, overlaps with H-1), 5.13 (t, 1H, $\left.J_{2,3} 10.2, J_{3,4} 9.6, \mathrm{H}-3\right), 4.88$ (dd, 1H, $\left.J_{2,3} 10.2, \mathrm{H}-2\right), 4.48$ (d, 1H, $J_{4,5}$ 9.9, H-5), 3.27 (s, 3H, N CH3), 2.41 (s, 3H, Ar $\mathrm{CH}_{3}$ ), 2.03, 2.00, 1.98 (each s, each 3H, each $\left.\mathrm{COCH}_{3}\right)$; selected ${ }^{1} \mathrm{H}$ NMR data for $\beta$-anomer: $\delta 5.02\left(\mathrm{t}, 1 \mathrm{H}, J_{2,3} 9.4, J_{3,4} 9.4, \mathrm{H}-3\right), 4.16(\mathrm{~d}, 1 \mathrm{H}$, $\left.J_{1,2} 8.8, \mathrm{H}-1\right), 4.01\left(\mathrm{~d}, 1 \mathrm{H}, J_{4,5} 9.9, \mathrm{H}-5\right) ;{ }^{13} \mathrm{C} \mathrm{NMR}$ for $\alpha$-anomer: $\left(75 \mathrm{MHz}, \mathrm{CDCl}_{3}\right): \delta 169.2$, 168.7, 167.7 (each s, each $\left.\mathrm{COCH}_{3}\right), 164.5\left(\mathrm{~s}, \mathrm{CONCH}_{3}\right), 138.5,137.6$ (each s, each $\left.\mathrm{Ar} \mathrm{C}\right), 129.5$, 126.0 (each d, each Ar C), 85.9 (d, C-1), 68.5, 65.9 (4 x d overlapping, C2-5), 36.9 (q, $\mathrm{N} \mathrm{CH}_{3}$ ), 20.1, 19.6, 19.6, 19.4 (each q, $3 \times \mathrm{COCH}_{3}$ and $\mathrm{Ar} \mathrm{CH}_{3}$ ); Selected ${ }^{13} \mathrm{C}$ NMR for $\beta$-anomer: 169.3, 167.9, 167.6 (each s, each $\left.\mathrm{COCH}_{3}\right), 163.2\left(\mathrm{~s}, \mathrm{CONCH}_{3}\right), 129.5,126.0$ (each d, each $\left.\mathrm{Ar} \mathrm{C}\right), 87.4$ (d, C-1), 71.6, 70.8, 69.1, 68.0 (each d, C2-5), 36.8 (q, $\left.\mathrm{N} \mathrm{CH}_{3}\right)$; FTIR $\left(\mathrm{CH}_{2} \mathrm{Cl}_{2}\right.$ solution on $\mathrm{NaCl}$ plates): $3649,3059,2962,2127,1756,1671,1514,1373,1257,1222,1208,1078,1057, \mathrm{~cm}^{-1}$; ES-HRMS: Found 449.1689, required 449.1672 [M+H] ${ }^{+}$.

\section{2,3,4-Tri-O-acetyl-1-azido-1-deoxy- $\alpha / \beta$-D-glucopyranuronic $\quad$ acid, $\quad N$-methyl- $p$ - methoxyphenylamide $\quad{ }^{1} \mathrm{H}$ NMR data for $\alpha$-anomer: $\left(300 \mathrm{MHz}, \mathrm{CDCl}_{3}\right): \delta 7.13(\mathrm{~m}, 2 \mathrm{H}, \mathrm{Ar}$}


Synthesis and structural analysis of anilides of glucuronic acid......Tosin, O'Brien et al. S10

H), $6.96(\mathrm{~d}, 2 \mathrm{H}, J$ 8.5, $\mathrm{Ar} \mathrm{H}), 5.50\left(\mathrm{~m}, 2 \mathrm{H}, \mathrm{H}-1\right.$ and H-3), $5.13\left(\mathrm{t}, 1 \mathrm{H}, J_{3,4} 9.9, J_{4,5} 9.7, \mathrm{H}-4\right), 4.86$ (dd, $\left.1 \mathrm{H}, J_{1,2} 4.3, J_{2,3} 10.2, \mathrm{H}-2\right), 4.43$ (d, $\left.1 \mathrm{H}, J_{4,5} 9.7, \mathrm{H}-5\right), 3.83$ (s, 3H, OCH $\left.H_{3}\right), 3.25$ (s, 3H, N $\mathrm{CH}_{3}$ ), 2.02, 2.01, 1.99 (each s, each $3 \mathrm{H}$, each $\mathrm{COCH}_{3}$ ); selected ${ }^{1} \mathrm{H}$ NMR data for $\beta$-anomer: $\delta 4.21\left(\mathrm{~d}, 1 \mathrm{H}, J_{1,2} 9.7, \mathrm{H}-5\right), 3.96\left(\mathrm{~d}, 1 \mathrm{H}, J_{4,5} 10.2, \mathrm{H}-5\right)$; FTIR $\left(\mathrm{CH}_{2} \mathrm{Cl}_{2}\right.$ solution on $\mathrm{NaCl}$ plates): $3440,2962,2840,2119,1753,1665,1512,1442,1369,1245,1041 \mathrm{~cm}^{-1} ;{ }^{13} \mathrm{C}$ NMR for $\alpha$-anomer: (75 MHz, $\left.\mathrm{CDCl}_{3}\right): \delta 169.2,168.7,167.8\left(\right.$ each s, each $\left.C \mathrm{COCH}_{3}\right), 163.4\left(\mathrm{~s}, \mathrm{CONCH}_{3}\right), 160.1,132.2$ (each s, each Ar C), 122.7, 118.5 (each d, each Ar C), 85.9 (d, C-1), 68.5, 68.4, 68.4, 66.0 (each d, $\mathrm{C} 2-5), 56.0$ (q, $\mathrm{Ar} \mathrm{OCH}_{3}$ ), 37.0 (q, $\mathrm{N} \mathrm{CH}_{3}$ ), 19.6, 19.5, 19.5 (each q, each $\mathrm{COCH}_{3}$ ); Selected ${ }^{13} \mathrm{C}$ NMR for $\beta$ - anomer: $\delta 163.0\left(\mathrm{~s}, \mathrm{CONCH}_{3}\right), 87.4(\mathrm{~d}, \mathrm{C}-1), 71.5,70.8,69.0,68.6$ (each d, C25); ES-HRMS: Found 465.1616, required $465.1622[\mathrm{M}+\mathrm{H}]^{+}$.

\section{2,3,4-Tri-O-acetyl-1-azido-1-deoxy- $\alpha / \beta$-D-glucopyranuronic acid, $N$-methyl- $p$-chloro}

phenylamide. ${ }^{1} \mathrm{H}$ NMR data for $\alpha$-anomer: $\left(300 \mathrm{MHz}, \mathrm{CDCl}_{3}\right): \delta 7.47(\mathrm{~d}, 2 \mathrm{H}, J$ 8.5, $\mathrm{Ar} \mathrm{H}), 7.18$ (d, 2H, $J$ 8.6, Ar H), 5.50 (d, $\left.1 \mathrm{H}, J_{1,2} 4.2, \mathrm{H}-1\right), 5.51$ (t, $1 \mathrm{H}, J_{3,4} 9.8, J_{4,5}, 9.9, \mathrm{H}-4$, overlaps with $\mathrm{H}-$ 1), 5.15 (t, $\left.1 \mathrm{H}, J_{2,3} 10.1, J_{3,4} 9.8, \mathrm{H}-3\right), 4.88\left(\mathrm{dd}, 1 \mathrm{H}, J_{2,3} 10.1, \mathrm{H}-2\right), 4.44$ (d, $\left.1 \mathrm{H}, J_{4,5} \mathrm{H}-5\right), 3.28$ (s, $3 \mathrm{H}, \mathrm{N} \mathrm{CH}$ ) $, 2.04,2.00,1.99$ (each s, each $3 \mathrm{H}$, each $\mathrm{COCH}_{3}$ ); selected ${ }^{1} \mathrm{H}$ NMR data for $\beta$ anomer: $\delta 5.04$ (t, $\left.1 \mathrm{H}, J_{2,3} 9.7, J_{3,4} 9.6, \mathrm{H}-3\right), 4.81\left(\mathrm{dd}, 1 \mathrm{H}, J_{1,2} 8.8, J_{2,3} 9.7, \mathrm{H}-2\right), 4.15$ (d, $1 \mathrm{H}, J_{1,2}$ 8.8, H-1), 3.96 (d, $\left.1 \mathrm{H}, J_{4,5} 9.8, \mathrm{H}-5\right) ;{ }^{13} \mathrm{C}$ NMR for $\alpha$-anomer: $\left(75 \mathrm{MHz}, \mathrm{CDCl}_{3}\right)$ : $\delta 169.2,168.8$, 167.6 (each s, each $\left.\mathrm{COCH}_{3}\right), 164.3\left(\mathrm{~s}, \mathrm{CONCH}_{3}\right), 139.6,133.6$ (each s, each $\left.\mathrm{Ar} \mathrm{C}\right), 129.1,127.7$ (each d, each Ar C), 85.9 (d, C-1), 68.5, 68.4, 68.4, 66.1 (each d, C2-5), 37.0 (q, $\mathrm{N} \mathrm{CH}_{3}$ ), 19.6, 19.5, (3 x q overlapping, each $\mathrm{COCH}_{3}$ ); Selected ${ }^{13} \mathrm{C}$ NMR for $\beta$-anomer: $129.1,127.8$ (each d, each $\mathrm{Ar}$ C), 87.4 (d, C-1), 71.4, 71.0, 67.9 (4 x d overlapping, C2-5); FTIR $\left(\mathrm{CH}_{2} \mathrm{Cl}_{2}\right.$ solution on 
Synthesis and structural analysis of anilides of glucuronic acid......Tosin, O'Brien et al. S11

$\mathrm{NaCl}$ plates): $3438,2962,2120,1755,1673,1492,1434,1368,1236,1044 \mathrm{~cm}^{-1}$; ES-HRMS: Found 469.1142, required 469.1126 [M+H] $]^{+}$

2,3,4-Tri-O-acetyl-1-azido-1-deoxy- $\alpha / \beta$-D-glucopyranuronic $\quad$ acid, $\quad N$-methyl- $\quad p$ -

fluorophenylamide. ${ }^{1} \mathrm{H}$ NMR data for $\alpha$-anomer: $\left(300 \mathrm{MHz}, \mathrm{CDCl}_{3}\right): \delta 7.29-7.13(\mathrm{~m}, 4 \mathrm{H}, \mathrm{Ar}$ H), $7.02\left(\mathrm{~m}, 2 \mathrm{H}\right.$, Ar H), 5.50 (d, 1H, $\left.J_{1,2} 4.2, \mathrm{H}-1\right), 5.50$ (t, 1H, H-4, overlaps with H-1), 5.15 (t, $\left.1 \mathrm{H}, J_{2,3} 10.2, J_{3,4} 9.6, \mathrm{H}-3\right), 4.88$ (dd, $\left.1 \mathrm{H}, J_{1,2} 4.2, J_{2,3} 10.2, \mathrm{H}-2\right), 4.42$ (d, 1H, $\left.J_{4,5} 9.7, \mathrm{H}-5\right), 3.27$ $\left(\mathrm{s}, 3 \mathrm{H}, \mathrm{N} \mathrm{CH}\right.$ ) $, 2.04,2.00,1.99$ (each s, each $3 \mathrm{H}$, each $\mathrm{COCH}_{3}$ ); selected ${ }^{1} \mathrm{H}$ NMR data for $\beta$ anomer: $\delta 5.04$ (t, 1H, $\left.J_{2,3} 9.4, J_{3,4} 9.7, \mathrm{H}-3\right), 4.13\left(\mathrm{~d}, 1 \mathrm{H}, J_{1,2} 8.8, \mathrm{H}-1\right), 3.95$ (d, 1H, $\left.J_{4,5} 9.9, \mathrm{H}-5\right)$;

${ }^{13} \mathrm{C}$ NMR for $\alpha$-anomer: (75 MHz, $\mathrm{CDCl}_{3}$ ): $\delta 169.3,168.8,167.67$ (each s, each $\left.\mathrm{COCH}_{3}\right), 164.4$ (s, $\mathrm{CONCH}_{3}$ ), 159.5 (s, Ar C), 137.1 (s, Ar C), 128.2, 128.1 (each d, each Ar C, J 8.8), 116.1, 115.8 (each d, each Ar C, J 22.9), 85.9 (d, C-1), 68.5, 68.4, 68.4, 66.0 (each d, C2-5), 37.1 (q, N $\mathrm{CH}_{3}$ ), 19.6, 19.6, 19.4 (each q, each $\mathrm{COCH}_{3}$ ); Selected ${ }^{13} \mathrm{C}$ NMR for $\beta$-anomer: 162.8 (s, $\mathrm{CONCH}_{3}$ ), 128.4 (s, Ar C), 116.0, 115.7 (each d, each Ar C, J 22.9), 87.4 (d, C-1), 71.6, 70.8, 69.0, 67.9 (each d, C2-5); FTIR $\left(\mathrm{CH}_{2} \mathrm{Cl}_{2}\right.$ solution on $\mathrm{NaCl}$ plates): 3480, 3069, 2967, 2119, 1915, 1756, 1661, 1559, 1510, 1366, 1221, 1155,1039, $978 \mathrm{~cm}^{-1}$; ES-HRMS: Found 453.1426, required $453.1426[\mathrm{M}+\mathrm{H}]^{+}$.

2,3,4-Tri- $O$-acetyl-1-azido-1-deoxy- $\alpha$-D-glucopyranuronic $\quad$ acid, $\quad N$-methyl- $p$ bromophenylamide. ${ }^{1} \mathrm{H}$ NMR data for $\alpha$-anomer: $\left(300 \mathrm{MHz}, \mathrm{CDCl}_{3}\right): \delta 7.60(\mathrm{~d}, 2 \mathrm{H}, J 8.6, \mathrm{Ar}$ H), $7.11(\mathrm{~d}, 2 \mathrm{H}, J$ 8.6, Ar H), 5.47 (d, 1H, H-1), 5.47 (t, 1H, H-4, overlaps with H-1), 5.14(t, 1H, $\left.J_{2,3} 10.1, J_{3,4} 9.6, \mathrm{H}-3\right), 4.86\left(\mathrm{dd}, 1 \mathrm{H}, J_{1,2} 4.3, J_{2,3} 10.1, \mathrm{H}-2\right), 4.42\left(\mathrm{~d}, 1 \mathrm{H}, J_{4,5} 9.9, \mathrm{H}-5\right), 3.27$ (s, $3 \mathrm{H}, \mathrm{N} \mathrm{CH}$ ), 2.03, 1.98, 1.97 (each s, each $3 \mathrm{H}$, each $\mathrm{COCH}_{3}$ ); selected ${ }^{1} \mathrm{H} \mathrm{NMR}$ data for $\beta$ - 
Synthesis and structural analysis of anilides of glucuronic acid......Tosin, O'Brien et al. S12

anomer: $\delta 5.03$ (t, $\left.1 \mathrm{H}, J_{2,3} 9.5, J_{3,4} 9.4, \mathrm{H}-3\right), 4.14$ (d, $\left.1 \mathrm{H}, J_{1,2} 8.6, \mathrm{H}-1\right), 3.95$ (d, $\left.1 \mathrm{H}, J_{4,5} 9.9, \mathrm{H}-5\right)$;

${ }^{13} \mathrm{C}$ NMR for $\alpha$-anomer: (75 MHz, $\mathrm{CDCl}_{3}$ ): $\delta 169.2,168.8,167.6$ (each s, each $\left.C \mathrm{COCH}_{3}\right), 164.2$ (s, $\mathrm{CONCH}_{3}$ ), 140.1 (s, Ar C), 132.2, 128.1 (each d, each Ar C), 121.5 (s, Ar C), 85.9 (d, C-1), 68.5, 68.4, 68.4, 66.1 (each d, C2-5), 37.0 (q, $\mathrm{N} \mathrm{CH}_{3}$ ), 19.6, 19.6, 19.5 (each q, each $\mathrm{COCH}_{3}$ ); Selected ${ }^{13} \mathrm{C}$ NMR for $\beta$-anomer: 132.1, 128.1 (each d, each Ar C), 87.4 (d, C-1), 71.4, 71.0, 68.0, 67.1 (each d, C2-5); FTIR ( $\mathrm{CH}_{2} \mathrm{Cl}_{2}$ solution on $\mathrm{NaCl}$ plates): $3446,2120,1756,1673,1486$, 1368, 1240, 1069, 1045, $1013 \mathrm{~cm}^{-1}$; ES-HRMS: Found 513.0643, required 513.0621 [M+H $]^{+}$.

\section{2,3,4-Tri- $O$-acetyl-1-azido-1-deoxy- $\alpha$-D-glucopyranuronic $\quad$ acid, $\quad N$-methyl- $p$ -}

methoxycarbonylphenylamide. $\quad{ }^{1} \mathrm{H}$ NMR data for $\alpha$-anomer: $\left(300 \mathrm{MHz}, \mathrm{CDCl}_{3}\right): \delta 8.12$ 7.26 (m (x2), 4H, Ar H), 5.49 (d, 1H, H-1), 5.49 (t, 1H, H-4, overlaps with H-1), 5.14 (t, 1H, H3), 4.48 (dd, 1H, H-2), 4.46 (d, 1H, J4,5 9.9, H-5), 3.95 (s, 3H, $\left.\mathrm{COCH}_{3}\right), 3.32$ (s, 3H, N CH $H_{3}$, 2.04, 2.00, 1.99 (each s, each 3H, each $\mathrm{COCH}_{3}$ ); selected ${ }^{1} \mathrm{H}$ NMR data for $\beta$-anomer: $\delta 5.06(\mathrm{t}$, 1H, H-3), 4.23 (d, 1H, $J_{4,5}$ 9.6, H-5); ${ }^{13} \mathrm{C}$ NMR for $\alpha$-anomer: $\left(75 \mathrm{MHz}, \mathrm{CDCl}_{3}\right)$ : $\delta 169.1,168.9,168.0\left(\right.$ each s, each $\left.\mathrm{COCH}_{3}\right), 166.2\left(\mathrm{~s}, \mathrm{COOCH}_{3}\right), 162.5\left(\mathrm{~s}, \mathrm{CONCH}_{3}\right), 144.0(\mathrm{~s}$, Ar C), 130.9 (d, Ar C), 126.2 (s, Ar C), 121.4 (d, Ar C), 85.9 (d, C-1), 68.5, 68.4, 68.3, 66.0 (each d, C2-5), $52.0\left(\mathrm{~s}, \mathrm{COOCH}_{3}\right), 37.0\left(\mathrm{~s}, \mathrm{~N} \mathrm{CH}_{3}\right), 19.6,19.6,19.4$ (each q, each $\mathrm{COCH}_{3}$ ); Selected ${ }^{13} \mathrm{C}$ NMR for $\beta$ - anomer: $\delta 166.2\left(\mathrm{~s}, \mathrm{COOCH}_{3}\right), 162.4\left(\mathrm{~s}, \mathrm{CONCH}_{3}\right), 87.4(\mathrm{~d}, \mathrm{C}-1), 71.4$, 70.4, 69.3, 68.4 (each d, C2-5); FTIR ( $\mathrm{CH}_{2} \mathrm{Cl}_{2}$ solution on $\mathrm{NaCl}$ plates): $3470,2956,2119,1755$, 1725, 1674, 1604, 1281, 1236, 1043, $1047 \mathrm{~cm}^{-1}$; ES-HRMS: Found 493.1579, required 493.1571 $[\mathrm{M}+\mathrm{H}]^{+}$. 
Analytical Data for 25g. ${ }^{2} \quad{ }^{1} \mathrm{H}-\mathrm{NMR}\left(300 \mathrm{MHz}, \mathrm{CDCl}_{3}\right): \delta 8.00(\mathrm{~s}, 1 \mathrm{H}, \mathrm{NH}), 7.48(\mathrm{dd}, 2 \mathrm{H}, J$ $8.5, J 1.1$, aromatic $\mathrm{H}), 7.33(\mathrm{t}, 2 \mathrm{H}, J 7.5$, aromatic $\mathrm{H}), 7.14(\mathrm{t}, 1 \mathrm{H}, J 7.5$, aromatic $\mathrm{H}), 5.77(\mathrm{~d}$, 1H, J 4.2, H-1), 5.49 (t, 1H, J 9.8, H-3), 5.22 (t, 1H, J4,5 10.0, H-4), 4.96 (dd, 1H, H-2), 4.50 (d, 1H, H-5), 2.12, 2.09, 2.03 (each s, each 3H, each $\left.\mathrm{COCH}_{3}\right) ;{ }^{13} \mathrm{C}-\mathrm{NMR}\left(\mathrm{CDCl}_{3}\right): \delta 170.3,169.9$, 169.8 (each s, each $\mathrm{COCH}_{3}$ ), 164.5 (s, CONH), 136.7 (s aromatic C), 129.3, 125.3, 120.7 (each d), 86.3 (d, C-1), 70.5, 70.3, 69.4, 69.0 (each d, C-2-5), 20.9, 20.8, 20.7 (each q, each $\mathrm{COCH}_{3}$ ); CI-HRMS: Found 421.1353, required $421.1359[\mathrm{M}+\mathrm{H}]^{+}$.

Analytical data for 31: ${ }^{1} \mathrm{H}-\mathrm{NMR}\left(300 \mathrm{MHz}, \mathrm{CDCl}_{3}\right): \delta 6.12\left(\right.$ br d, $\left.1 \mathrm{H}, J_{\mathrm{CH}-\mathrm{N} H} 7.9, \mathrm{NH}\right)$, 5.54 (apt t, 1H, J 9.8, H-3), 5.44 (d, 1H, $\left.J_{1,2} 3.3, \mathrm{H}-1\right), 5.06$ (dd, 1H, $\left.J_{4,5} 10.2, \mathrm{H}-4\right), 4.76$ (dd, 1H, H-2), $4.29(\mathrm{~d}, 1 \mathrm{H}, \mathrm{H}-5), 4.03\left(\mathrm{~m}, 1 \mathrm{H}, \mathrm{CH}\left(\mathrm{CH}_{3}\right)_{2}\right), 2.11,2.07,2.02$ (each s, each 3H, each $\left.\mathrm{COCH}_{3}\right), 1.18$ and $1.16(2 \mathrm{~d}$, each $3 \mathrm{H}) ;{ }^{13} \mathrm{C}-\mathrm{NMR}\left(\mathrm{CDCl}_{3}\right): \delta 170.3,169.9,169.8$ (each s, each $\left.\mathrm{COCH}_{3}\right), 165.7(\mathrm{~s}, \mathrm{CONH}), 86.3$ (d, C-1), 70.4, 70.3, 69.5, 69.0 (each d, C-2-5), 41.5 (d, $\left.\mathrm{CH}\left(\mathrm{CH}_{3}\right)_{2}\right)$, 22.7, 22.6 (each q, each $\left.\mathrm{CH}\left(\mathrm{CH}_{3}\right)_{2}\right), 20.9,20.8,20.7$ (each q, each $\mathrm{COCH}_{3}$ ); CIHRMS: Found 387.1511, required 387.1516 [M+H] $]^{+}$

Analytical data for 32. ${ }^{1} \mathrm{H}-\mathrm{NMR}\left(300 \mathrm{MHz}, \mathrm{CDCl}_{3}\right): \delta 7.49-7.43(\mathrm{~m}, 3 \mathrm{H}$, aromatic $\mathrm{H})$, 7.34-7.23 (m, 2H, aromatic H), $5.54\left(\mathrm{~d}, 1 \mathrm{H}, J_{1,2} 4.5, \mathrm{H}-1\right), 5.52$ (t, 1H, H-4), $5.16(\mathrm{t}, 1 \mathrm{H}, J$ 10.0, H-3), 4.92 (dd, 1H, H-2), 4.48 (d, 1H, J 9.8, H-5), 3.30 (s, 3H, OCH $H_{3}$, 2.03, 2.01, 1.99 (each s, each $3 \mathrm{H}$, each $\mathrm{COCH} 3) ;{ }^{13} \mathrm{C}-\mathrm{NMR}\left(\mathrm{CDCl}_{3}\right): \delta 170.5,170.0,168.9$ (each s, each $\left.\mathrm{COCH}_{3}\right), 165.7$ (s, CONMePh), 142.5 (s, aromatic C), 130.2, 128.8, 127.5 (each d), 87.3 (d, C-1), 69.9, 69.8,

\footnotetext{
${ }^{2}$ Tosin, M.; Murphy, P.V. Org. Lett. 2002, 4, 3675.
} 
Synthesis and structural analysis of anilides of glucuronic acid......Tosin, O'Brien et al. S14

69.7, 67.3 (each d, C-2-5), 38.2 (q, $\mathrm{NCH}_{3} \mathrm{Ph}$ ), 20.9 (two q, overlapping, each $\mathrm{COCH}_{3}$ ), 20.7 (q, $\mathrm{COCH}_{3}$ ); CI-HRMS: Found 435.1512, required 435.1516 [M+H] $]^{+}$

\section{General method for deacetylation}

Sodium methoxide (freshly prepared 1.0 M solution in $\mathrm{MeOH}, 0.01 \mathrm{~mL}$ ) was added to a solution of the reactant in methanol $(0.1 \mathrm{~g} / 10 \mathrm{~mL})$ at $0^{\circ} \mathrm{C}$ and left to stir for $1 \mathrm{~h}$. The solvent was then removed and the resulting residue dissolved in water. The solution was acidified to $\mathrm{pH} 5-6$ by the addition of Amberlite IR-120 $\left(\mathrm{H}^{+}\right)$and filtration and freeze-drying afforded the desired compounds. The different anomers were, where possible, separated by preparative HPLC (YMCPack C-4 reverse phase, column , S-5 $\mu \mathrm{m}, 20 \mathrm{~nm}, 250 \times 20 \mathrm{~mm}$ ), isocratic elution $\mathrm{H}_{2} \mathrm{O} / \mathrm{CH}_{3} \mathrm{CN}$ 70:30, flow rate $10 \mathrm{~mL} / \mathrm{min})$. 


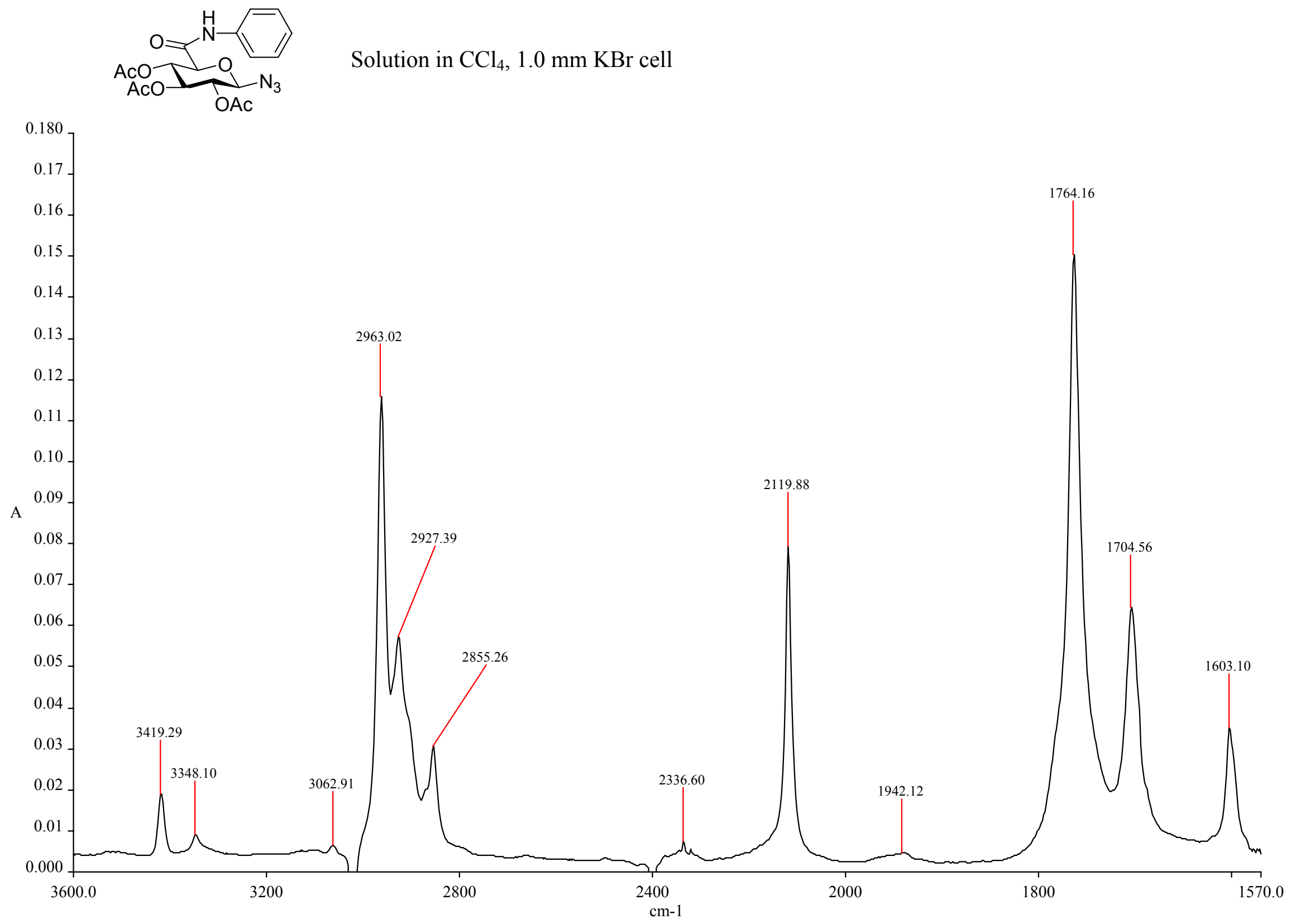




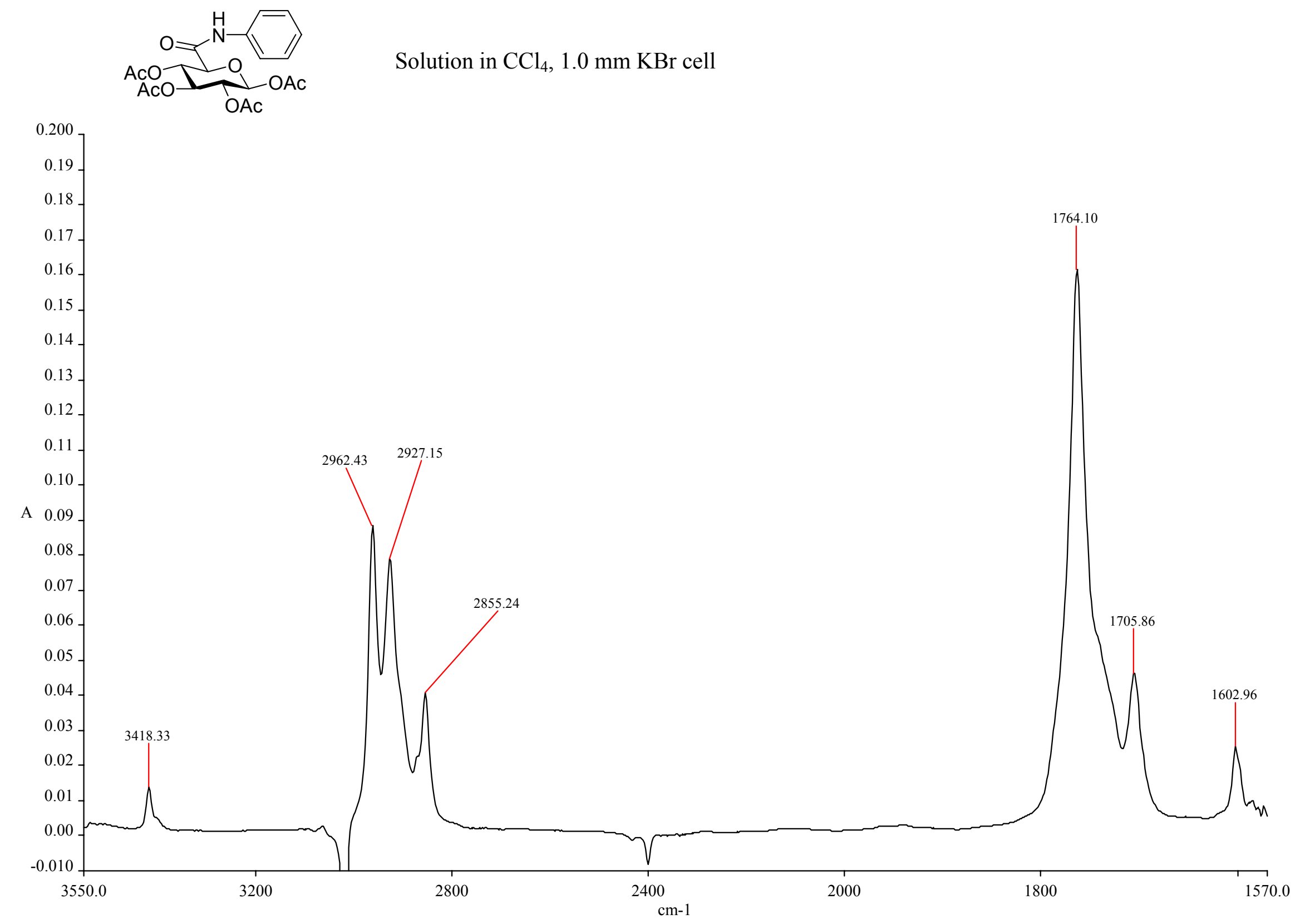




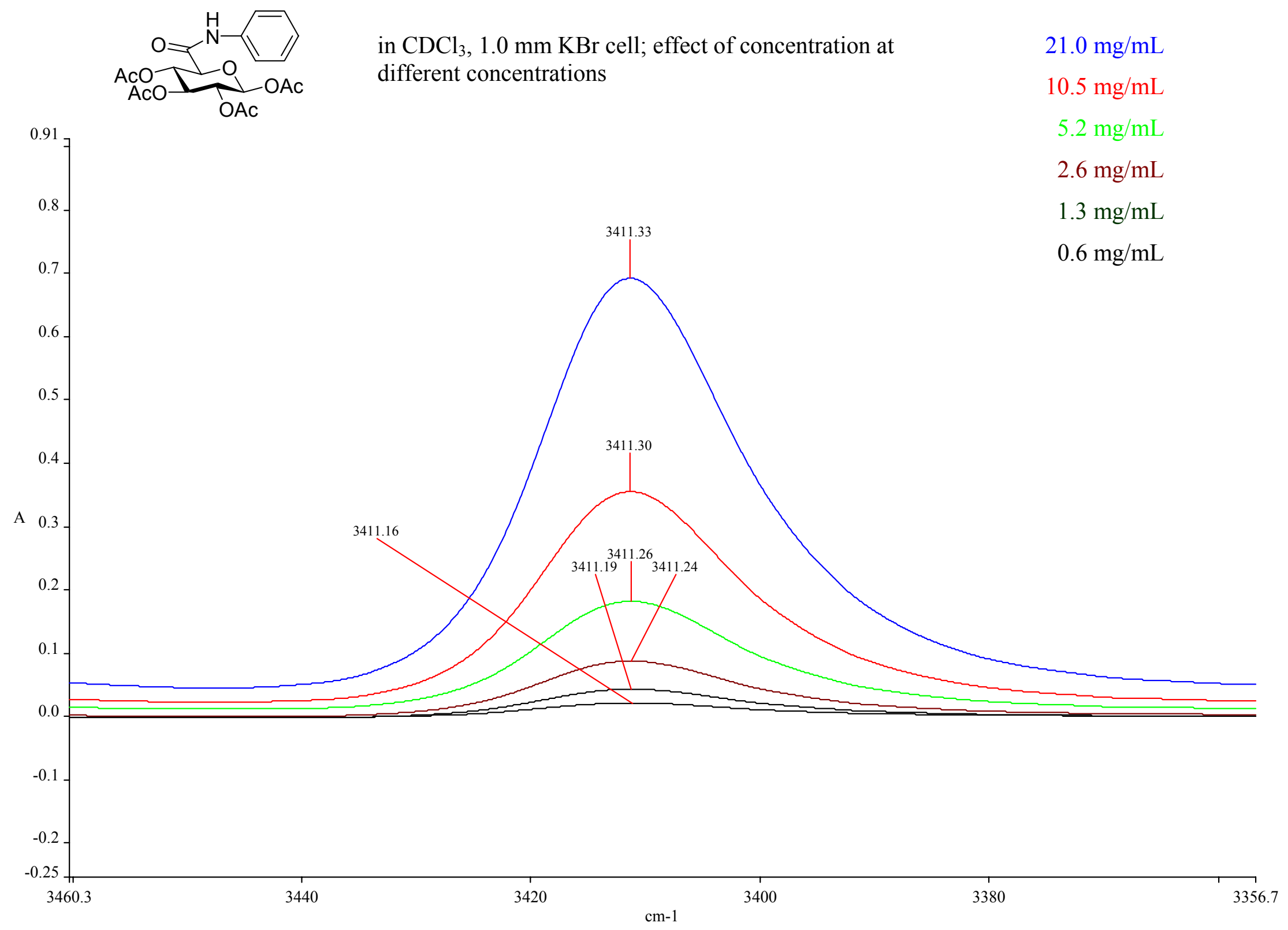




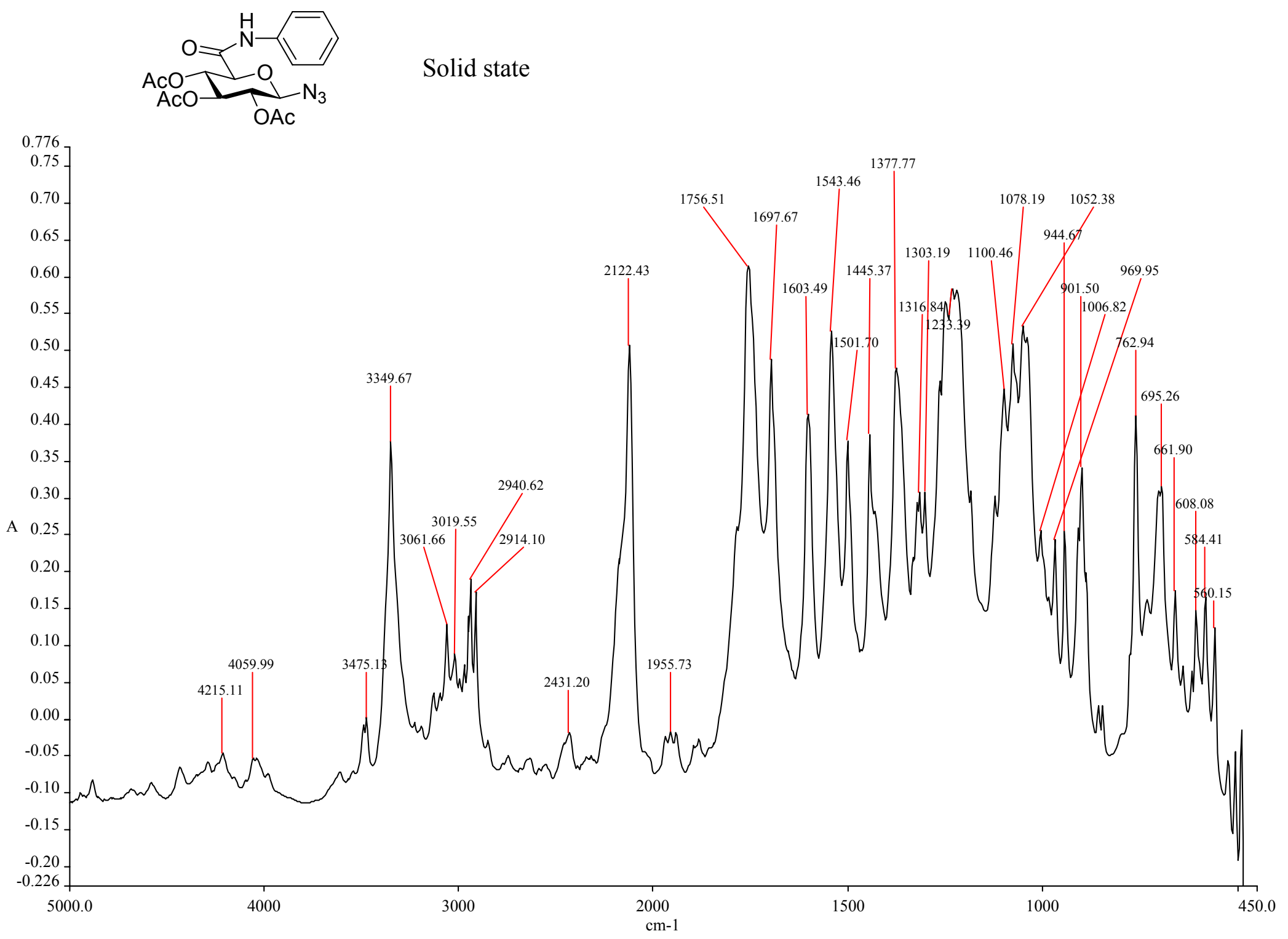


${ }^{1} \mathrm{H}-\mathrm{NMR}$ of<smiles>CC(C)Nc1ccc(Br)cc1</smiles>

13

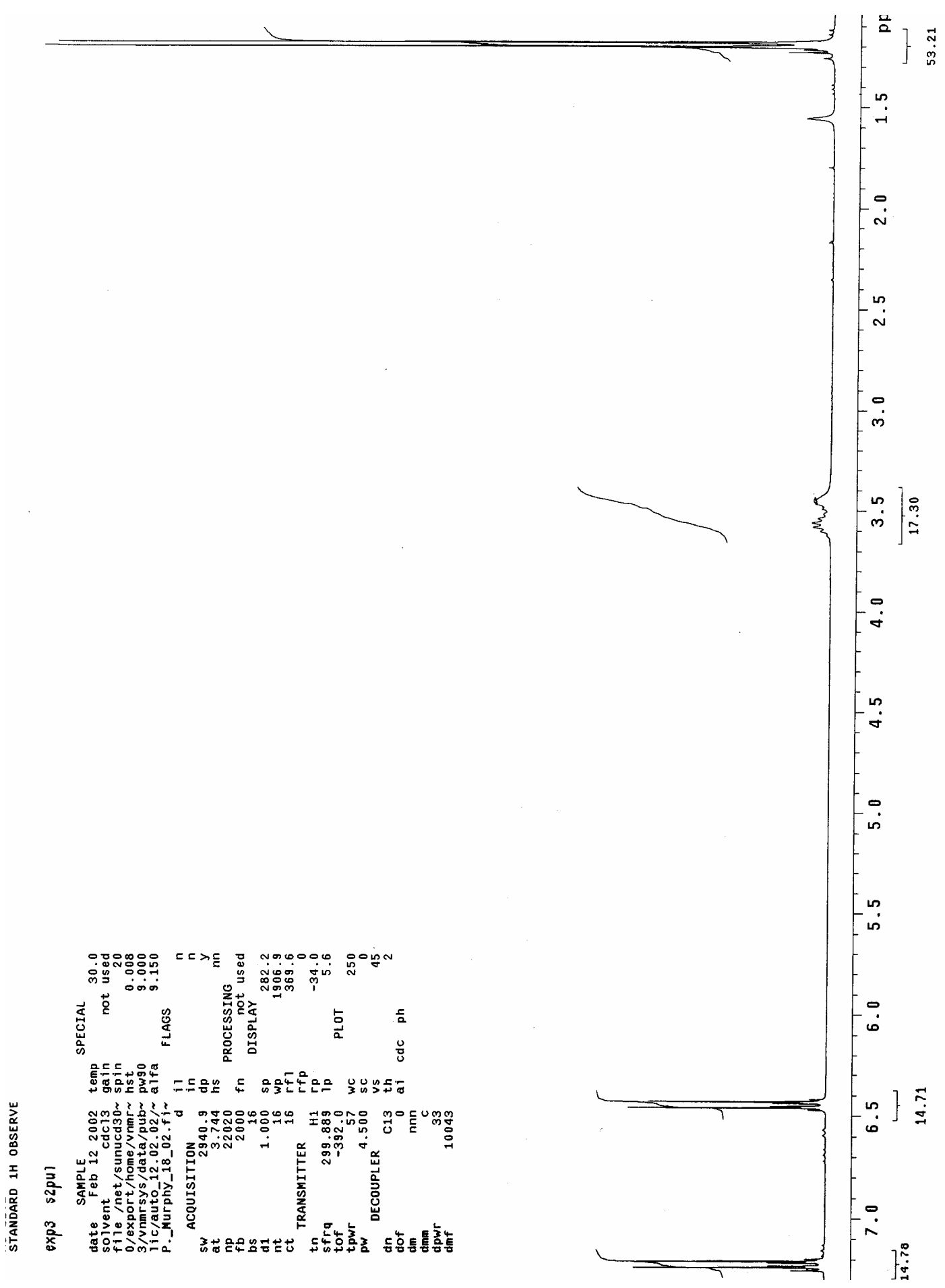


${ }^{1} \mathrm{H}-\mathrm{NMR}$ of
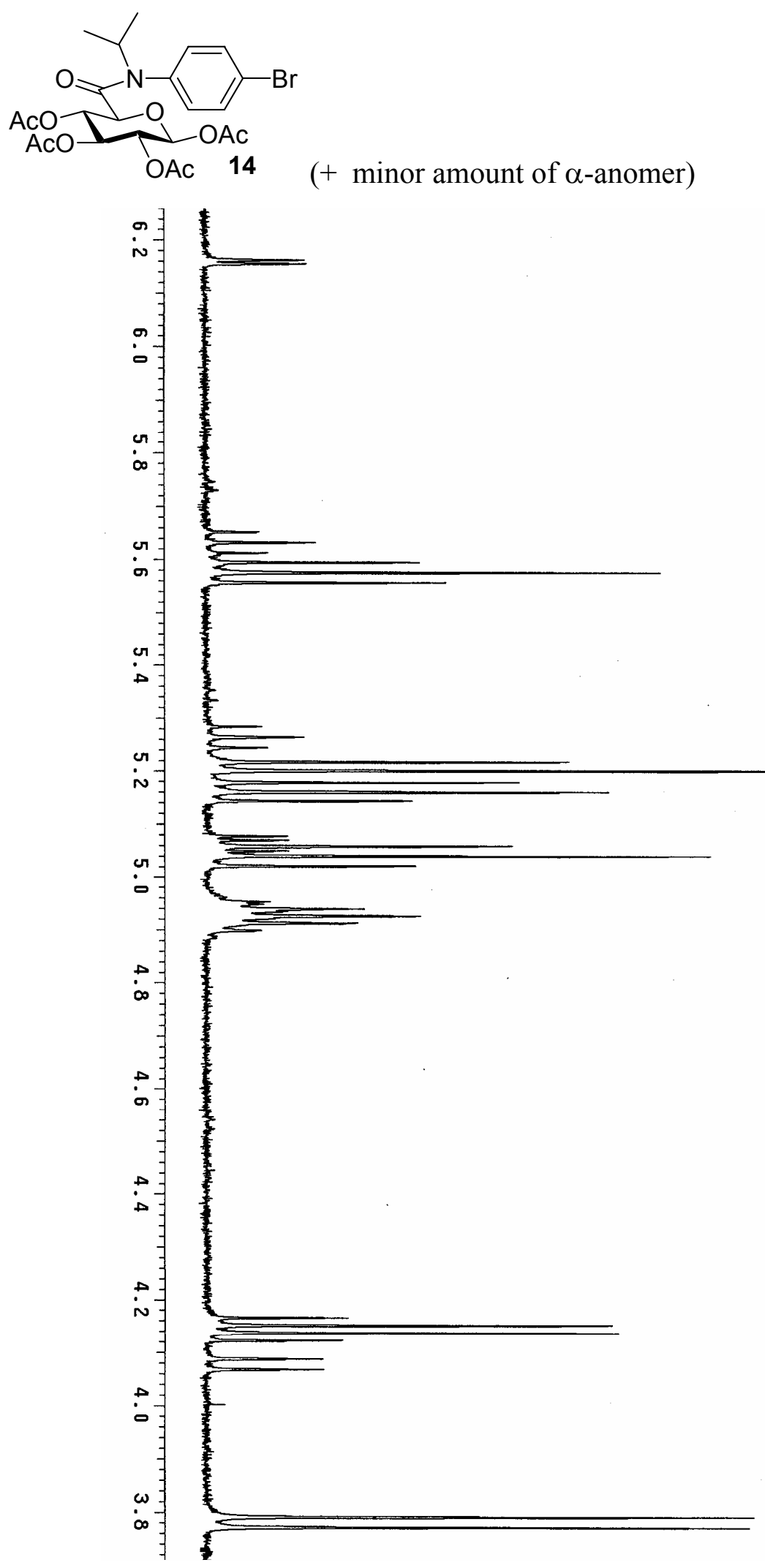


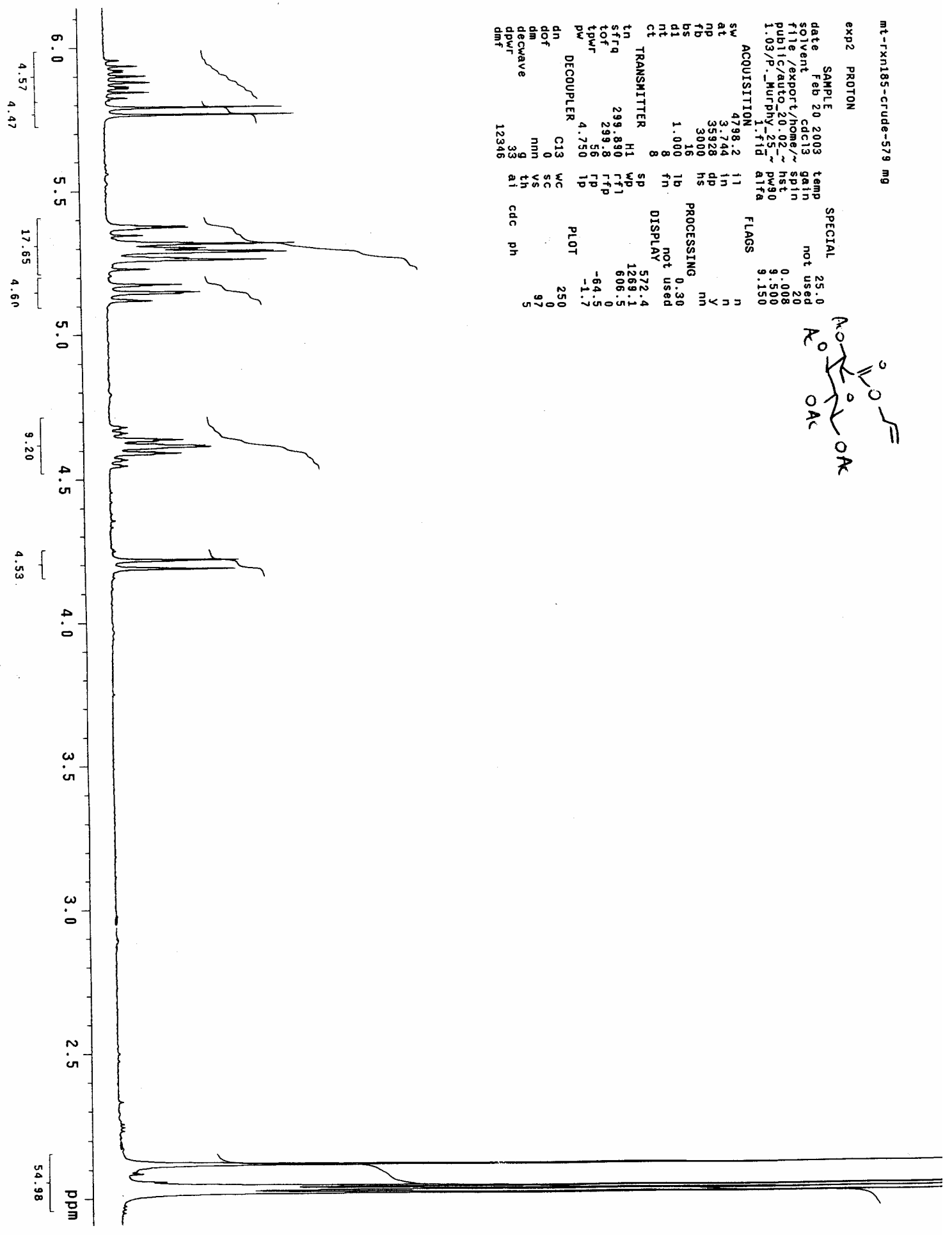


${ }^{1} \mathrm{H}-\mathrm{NMR}$ of $\mathbf{1 6}$

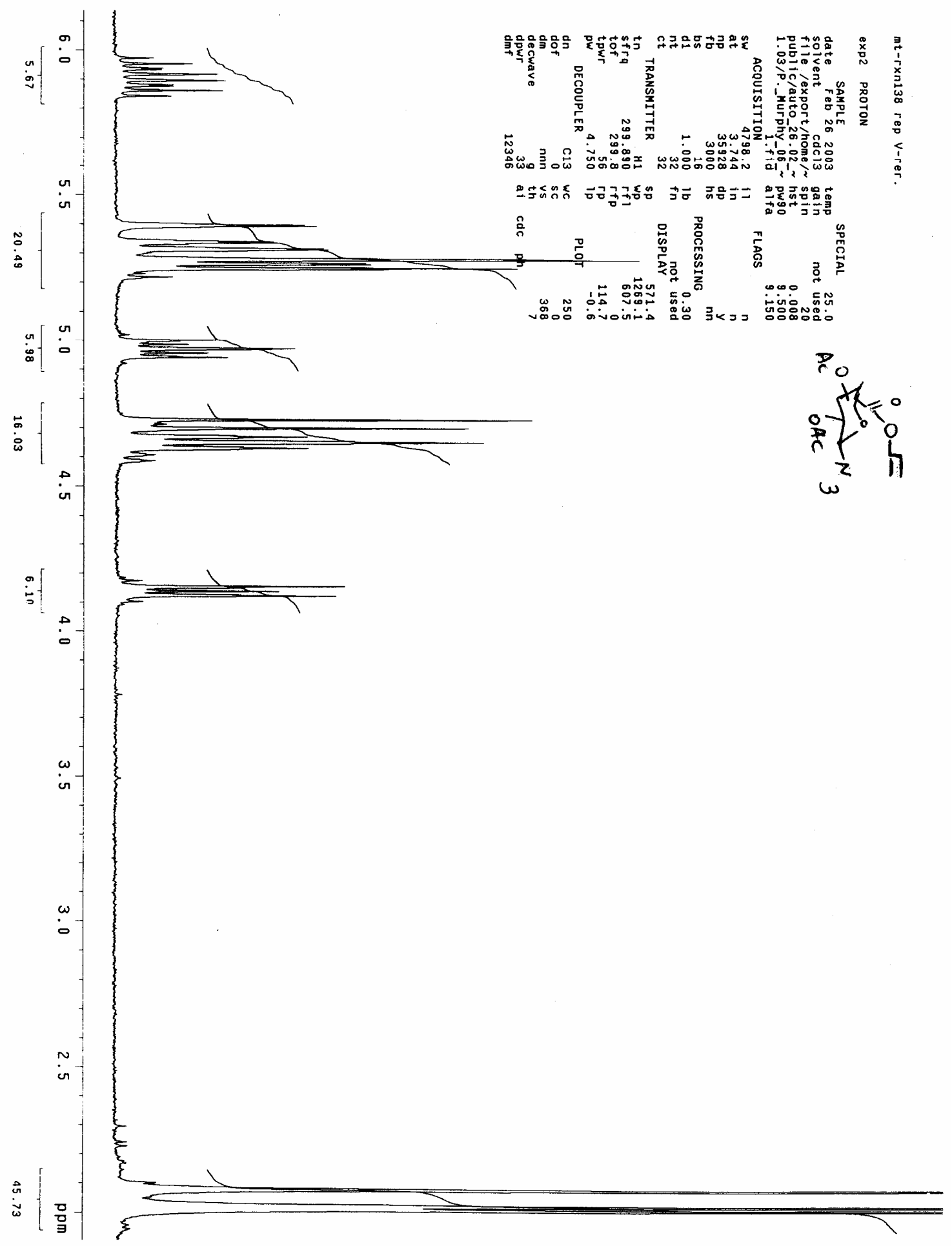



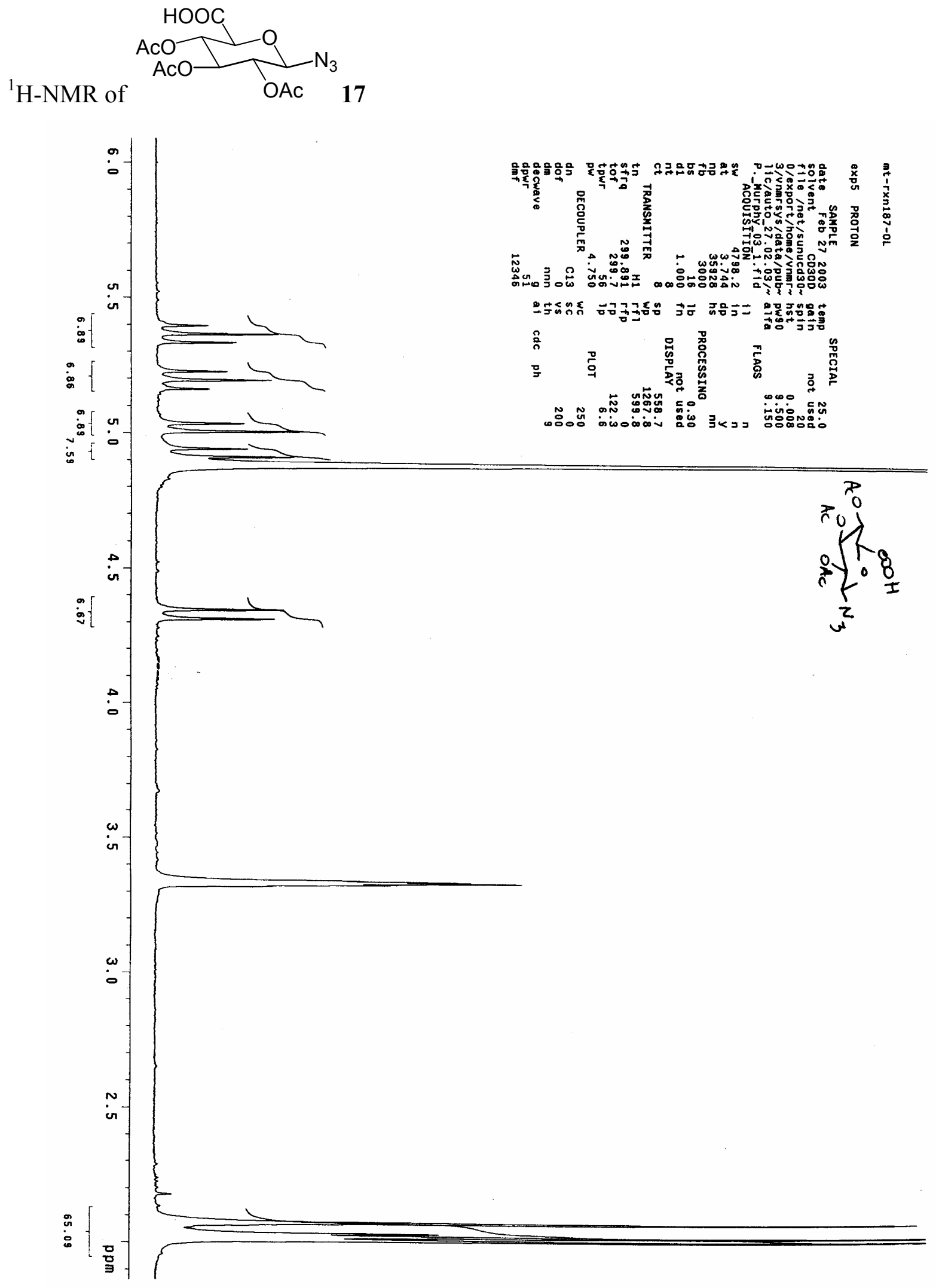
${ }^{1} \mathrm{H}-\mathrm{NMR}$ of

$\mathrm{OAc}_{20}$

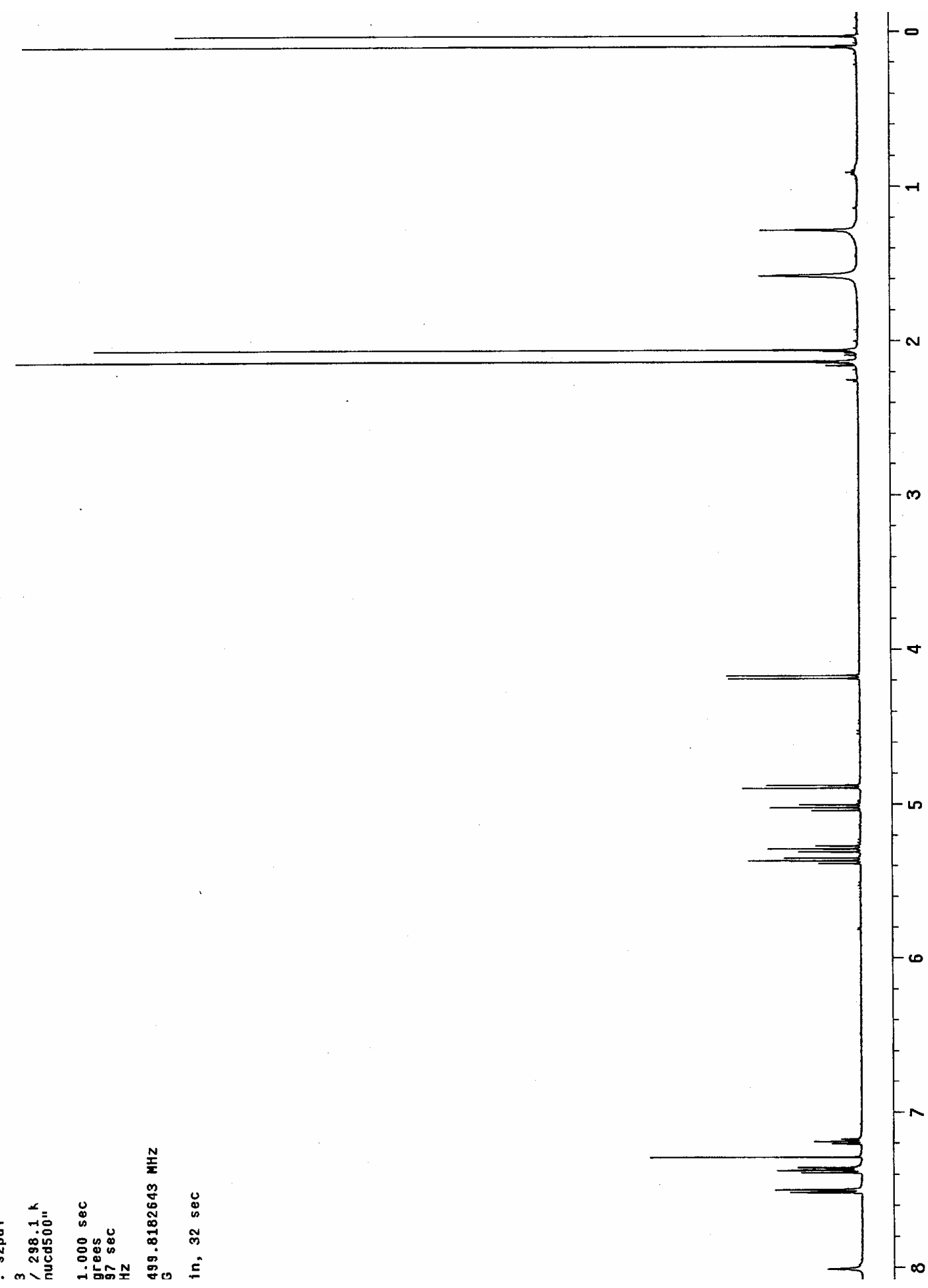



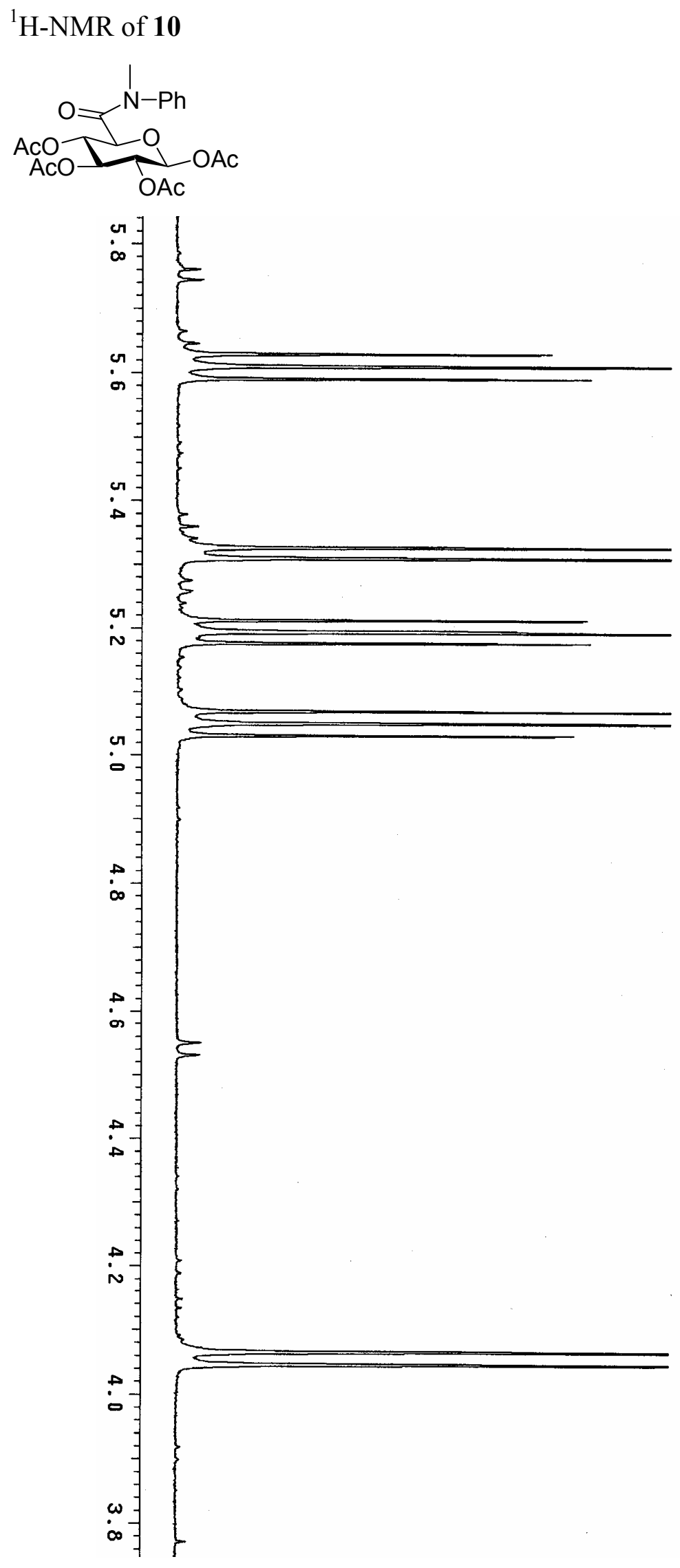
${ }^{1} \mathrm{H}-\mathrm{NMR}$ of $\mathbf{2 4 a}$ and $25 \mathrm{a}$

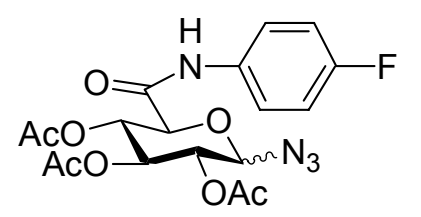

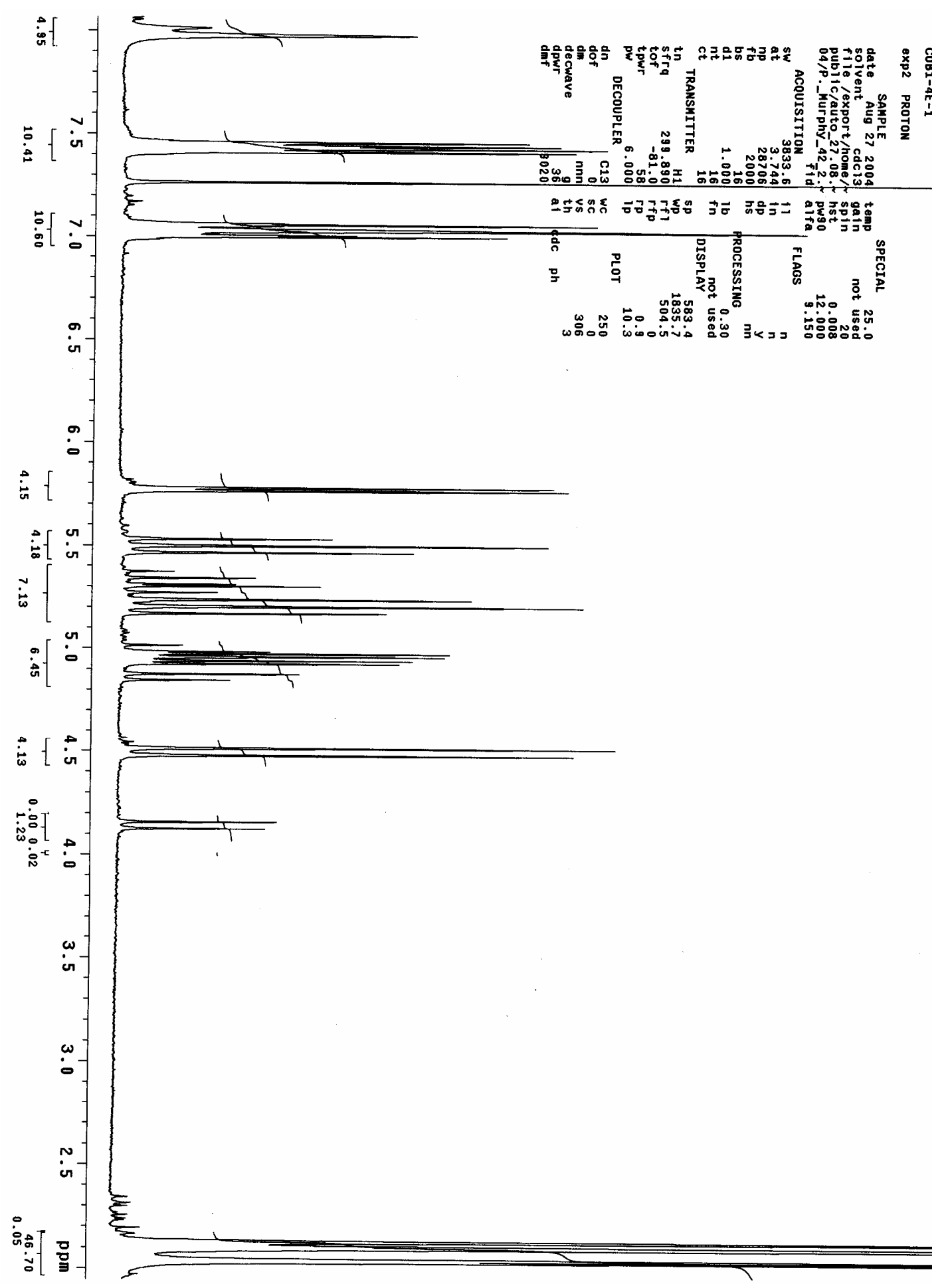


${ }^{1} \mathrm{H}-\mathrm{NMR}$ of $\mathbf{2 4 b}$ and $\mathbf{2 5 b}$

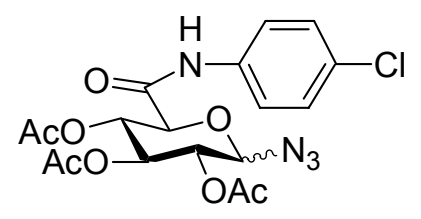

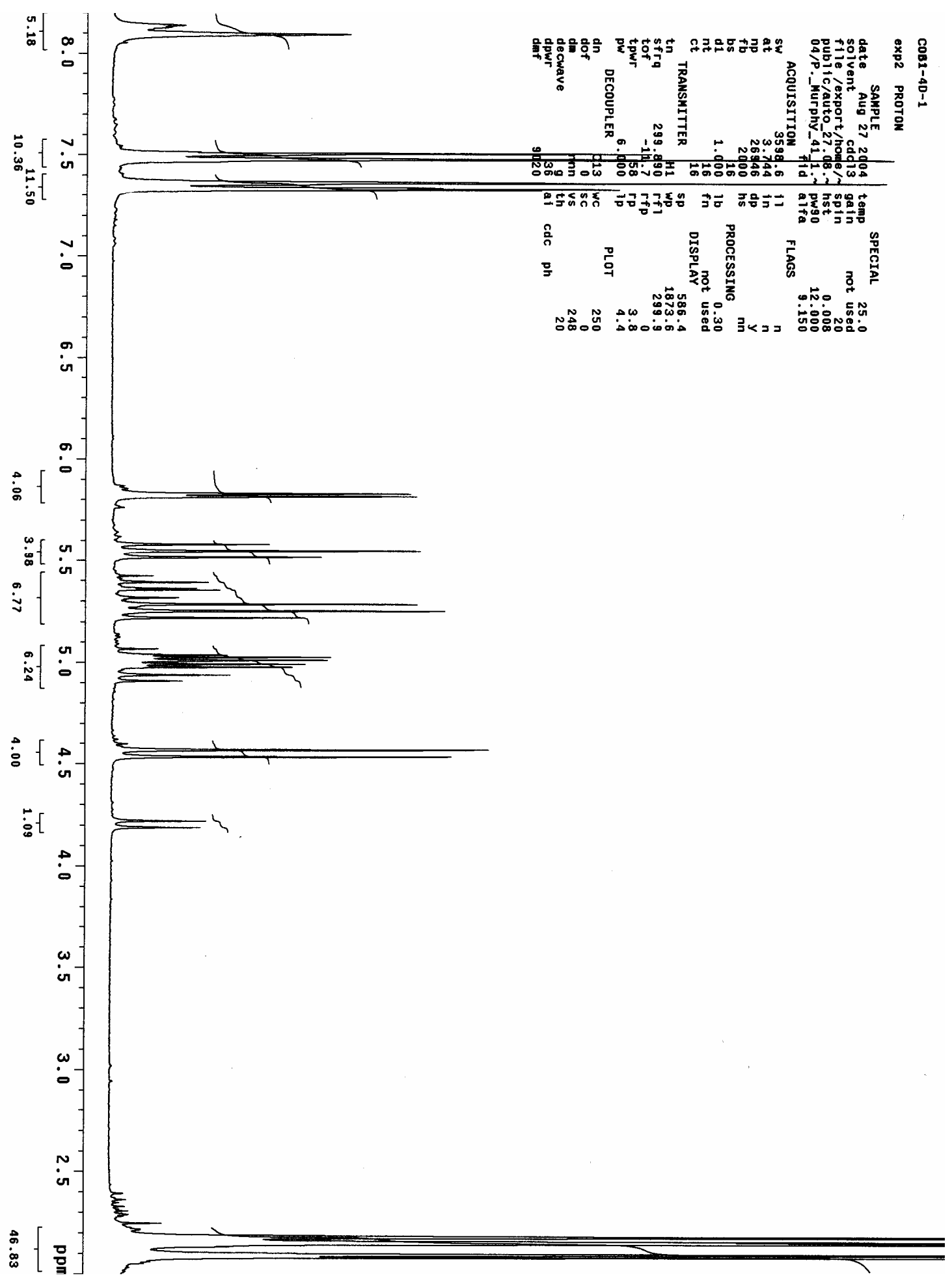


${ }^{1} \mathrm{H}-\mathrm{NMR}$ of $24 \mathrm{c}$ and $25 \mathrm{c}$

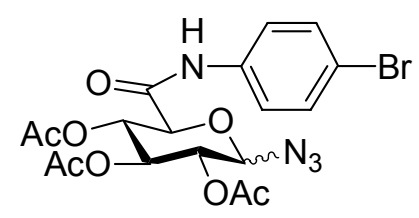

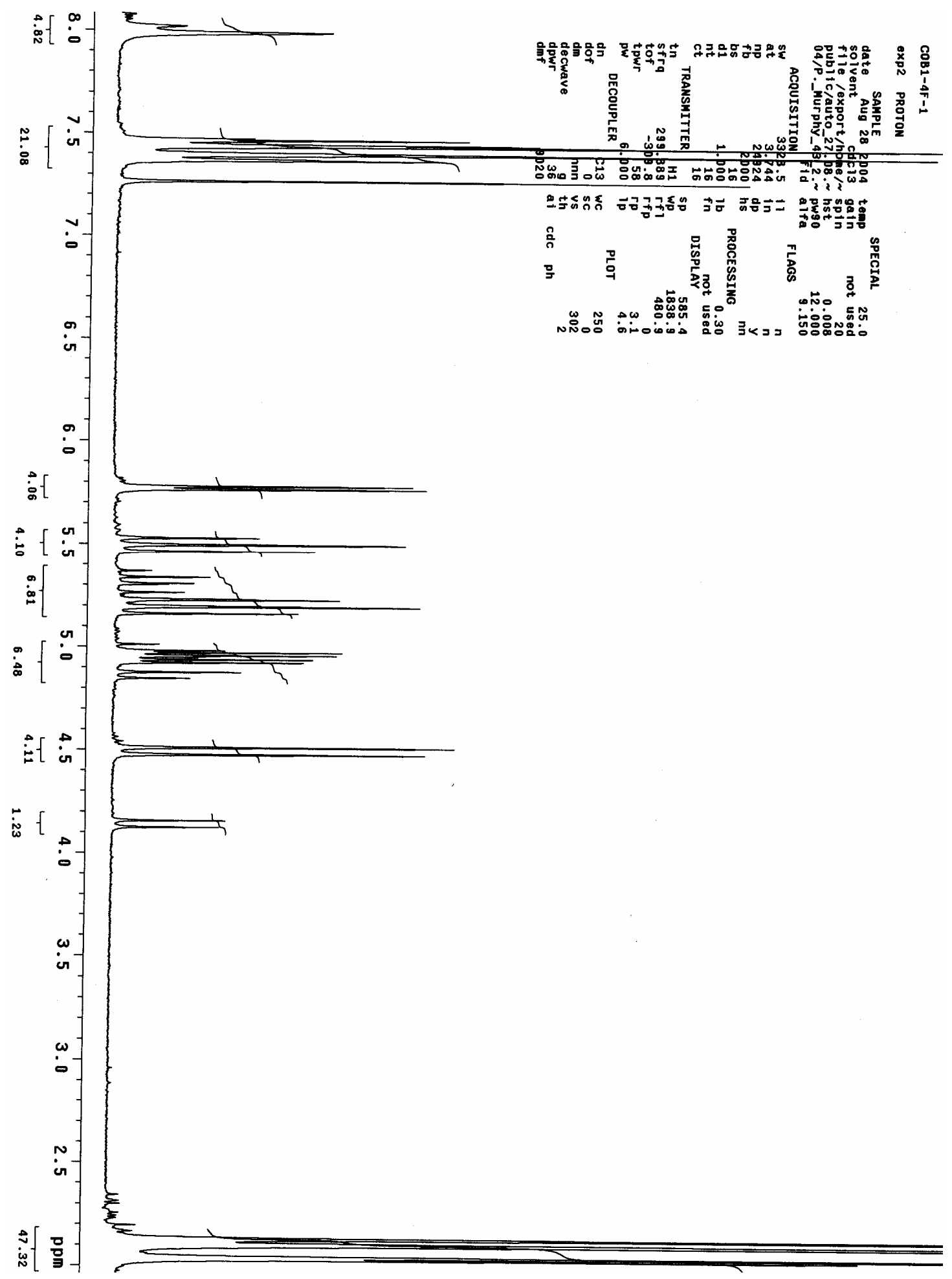


${ }^{1} \mathrm{H}-\mathrm{NMR}$ of $\mathbf{2 4 d}$ and $\mathbf{2 5 d}$

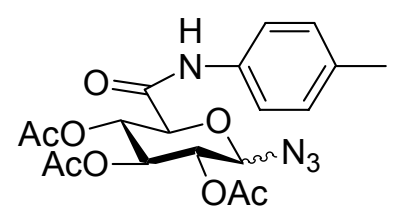

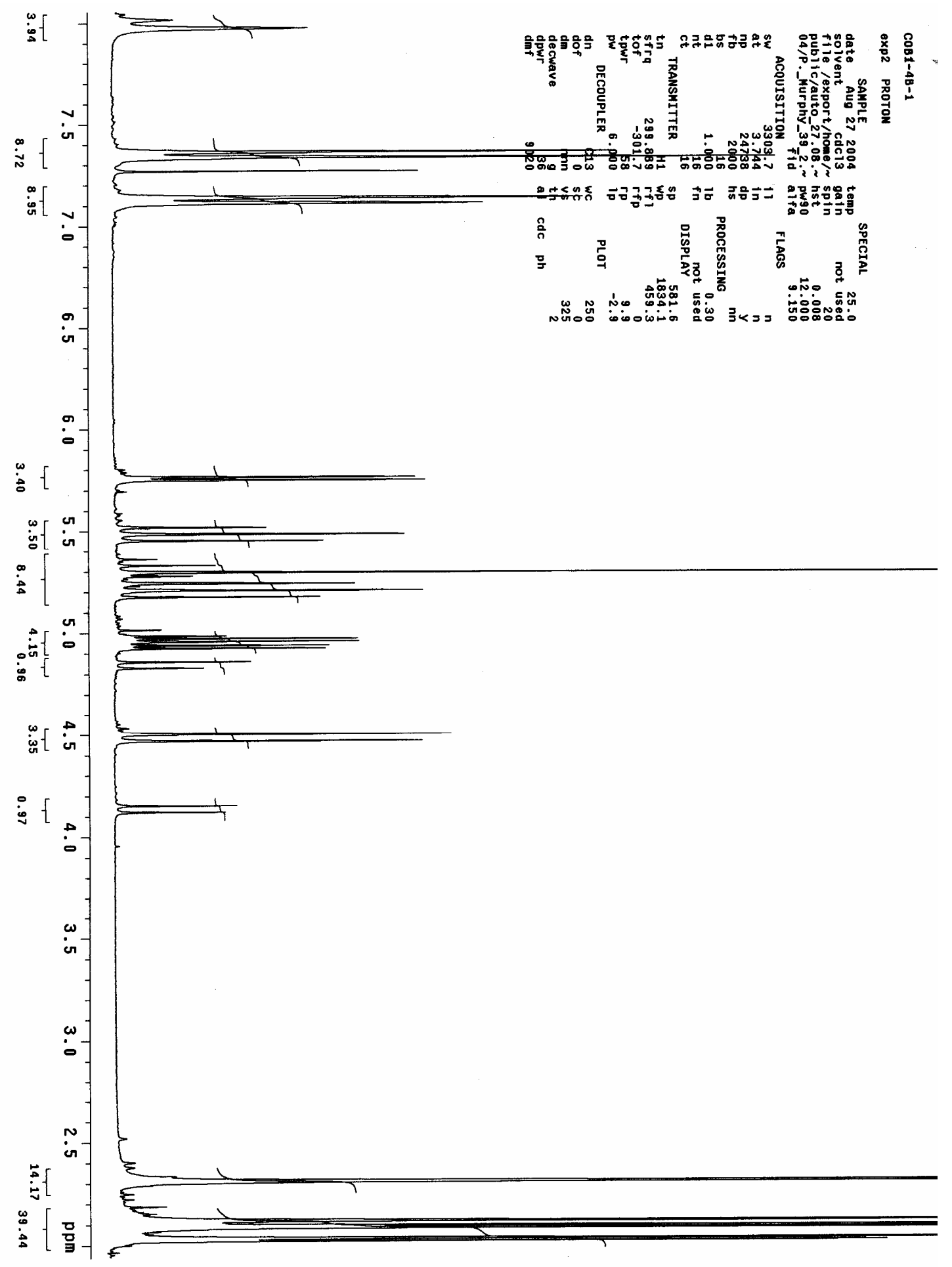


${ }^{1} \mathrm{H}-\mathrm{NMR}$ of $24 \mathrm{e}$ and $25 \mathrm{e}$

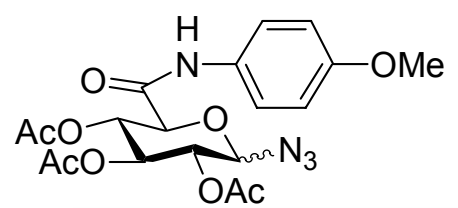

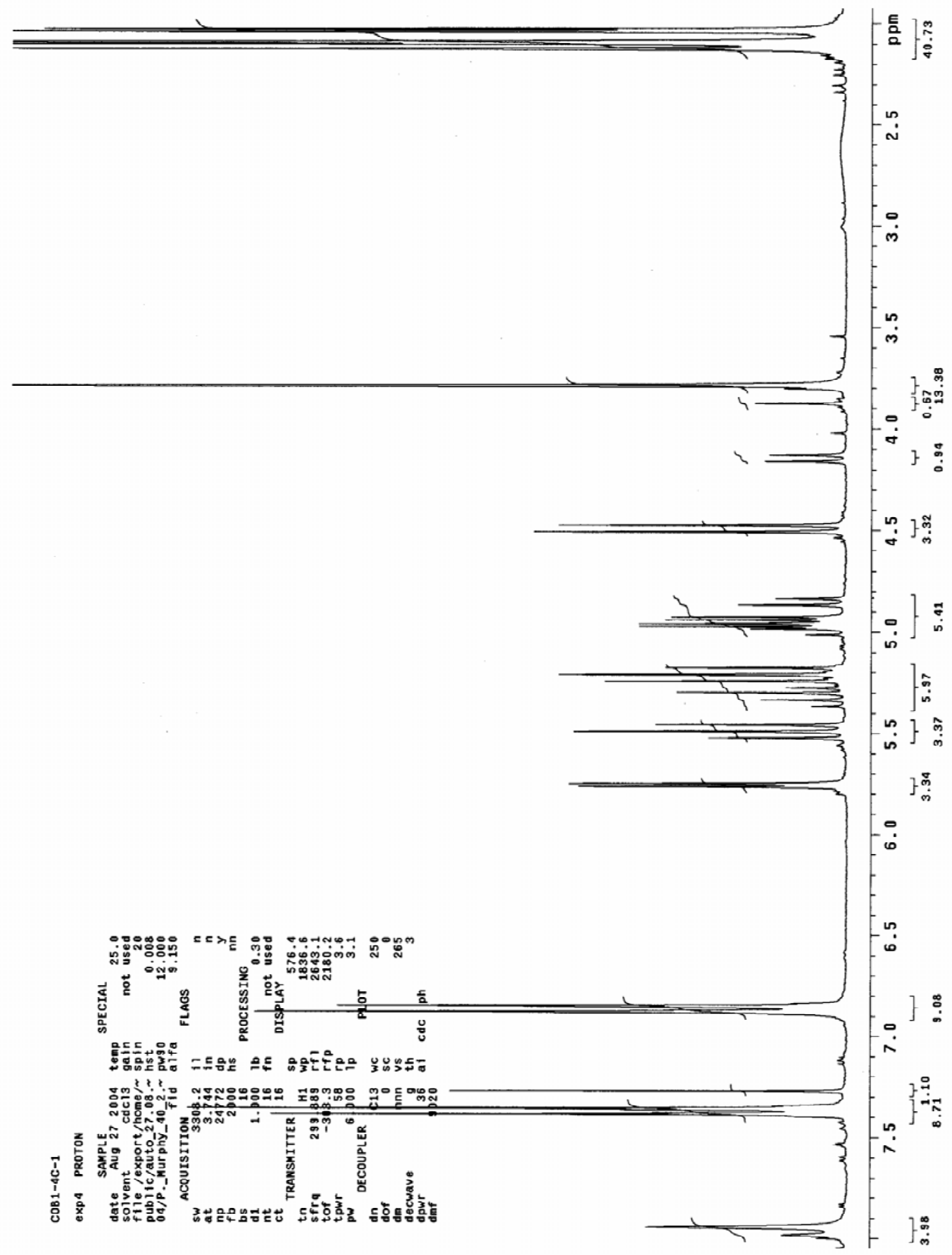


${ }^{1} \mathrm{H}-\mathrm{NMR}$ of $\mathbf{2 4 f}$ and $\mathbf{2 5 f}$

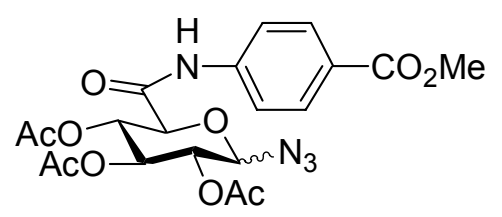

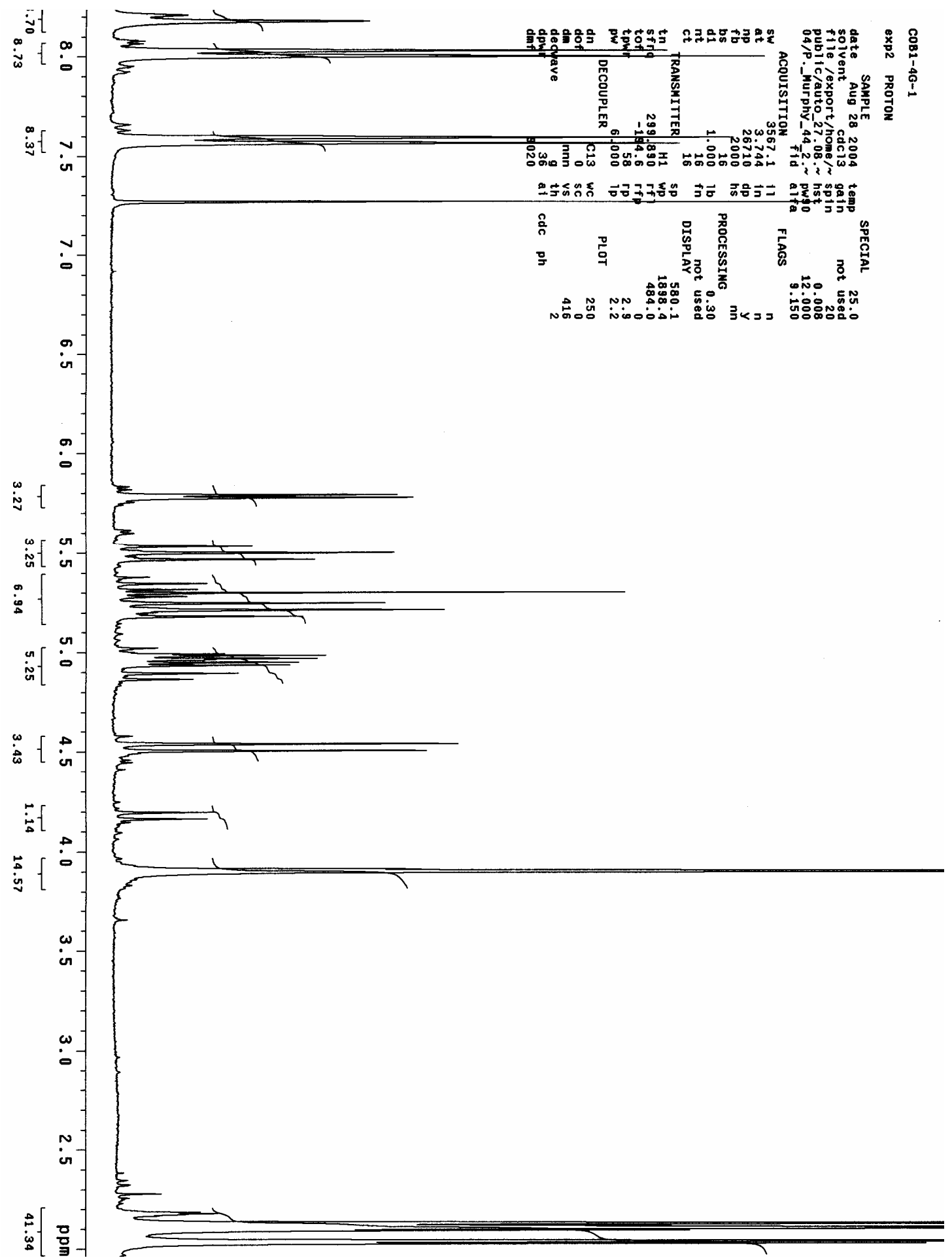



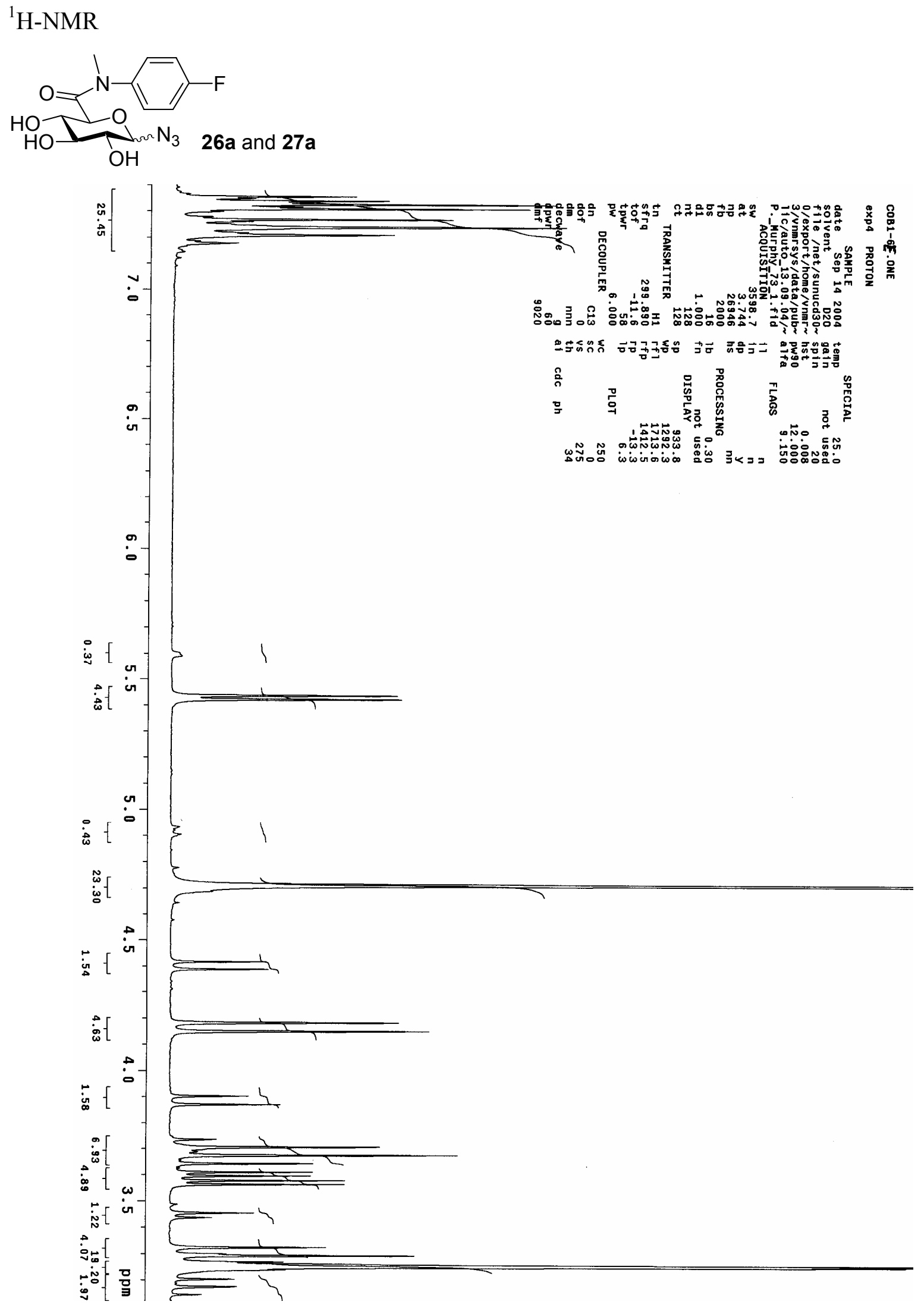
${ }^{1} \mathrm{H}-\mathrm{NMR}$

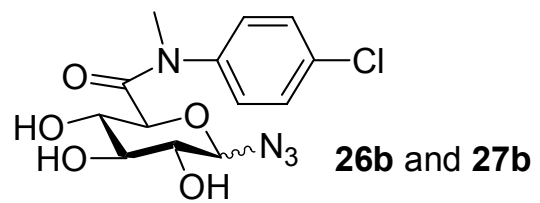

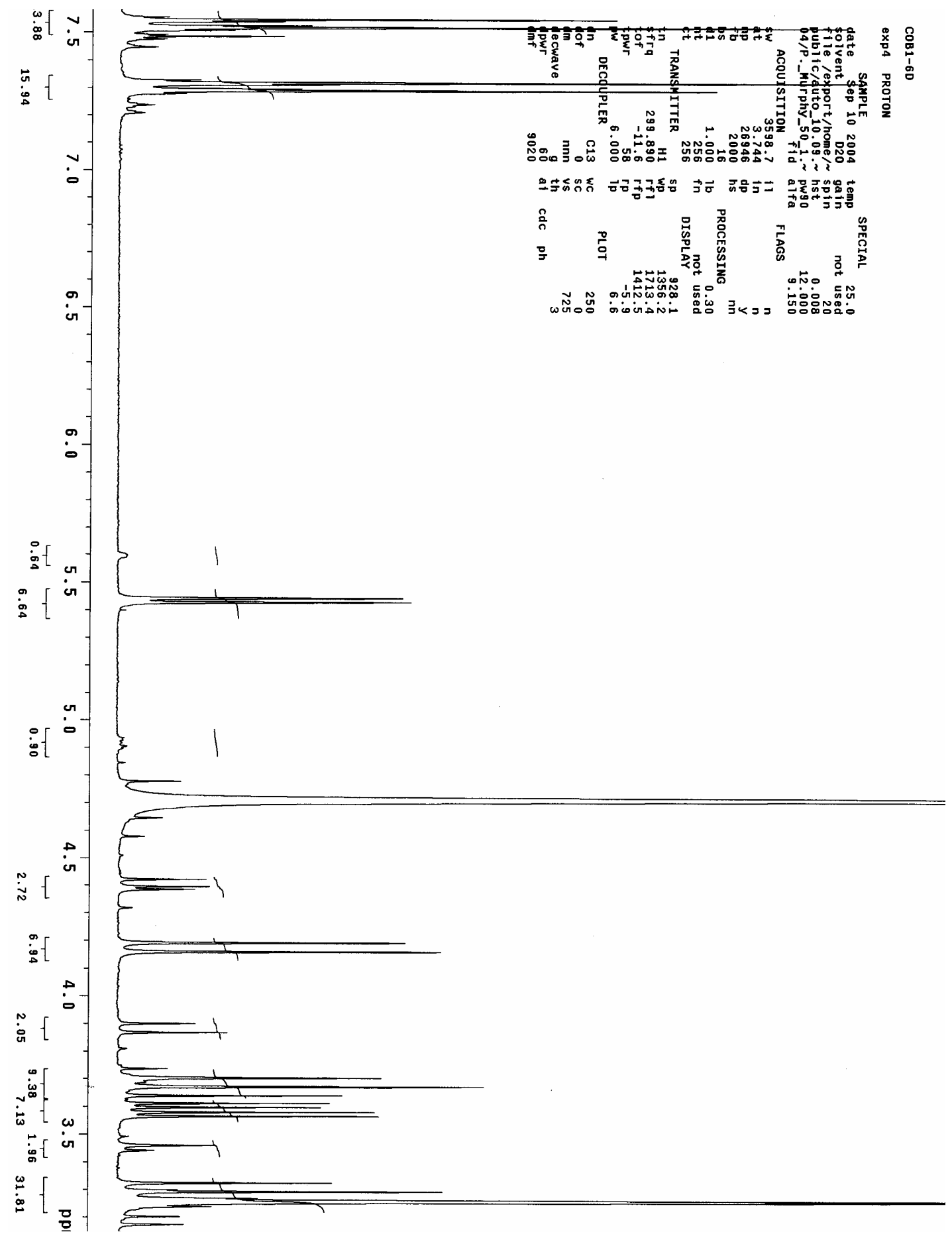


${ }^{1} \mathrm{H}-\mathrm{NMR}$

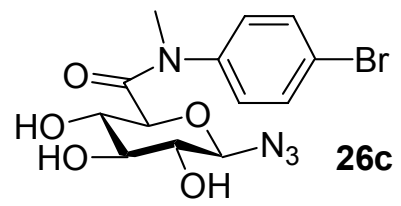

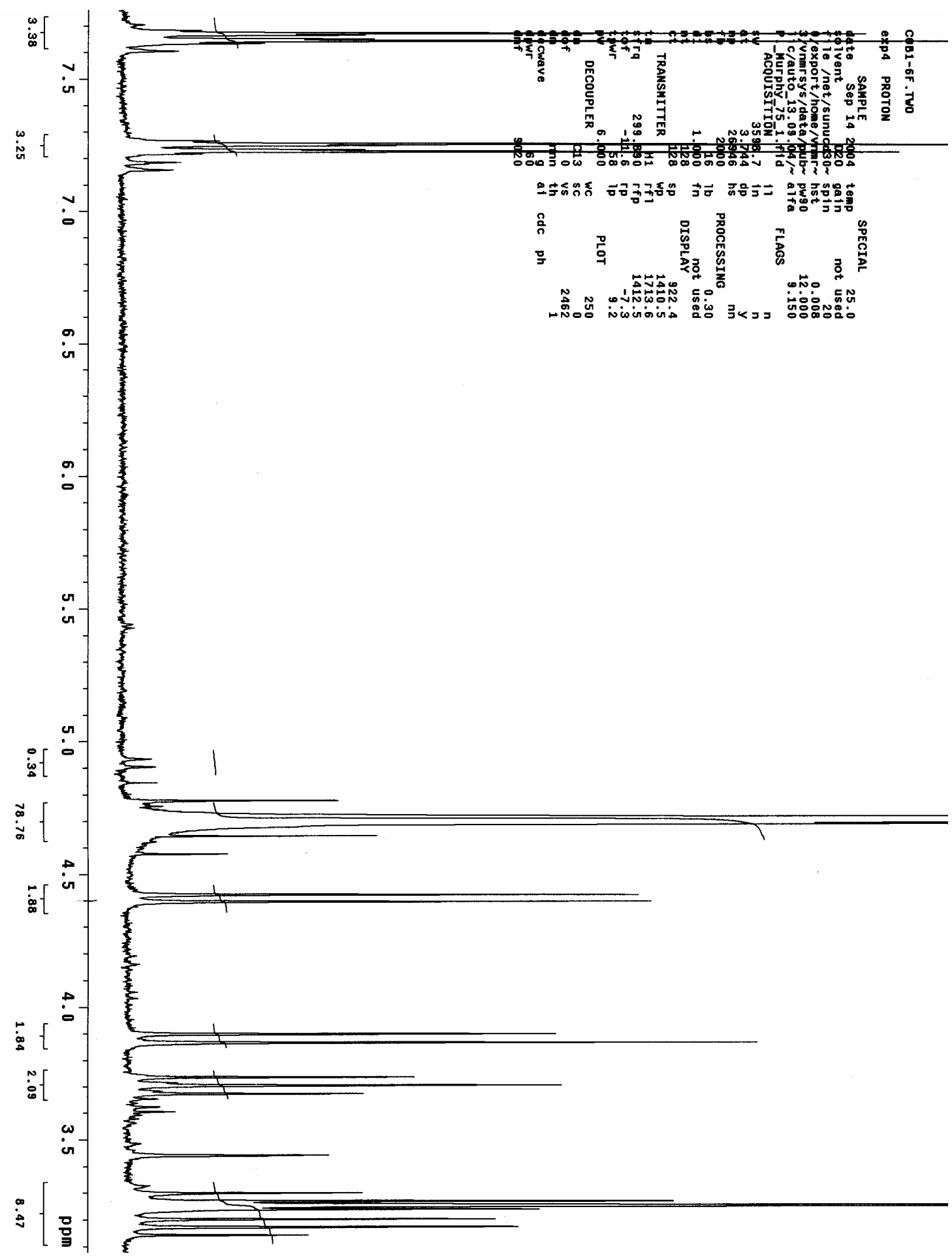


${ }^{1} \mathrm{H}-\mathrm{NMR}$

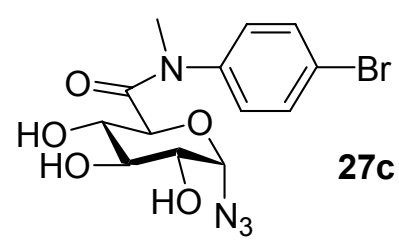

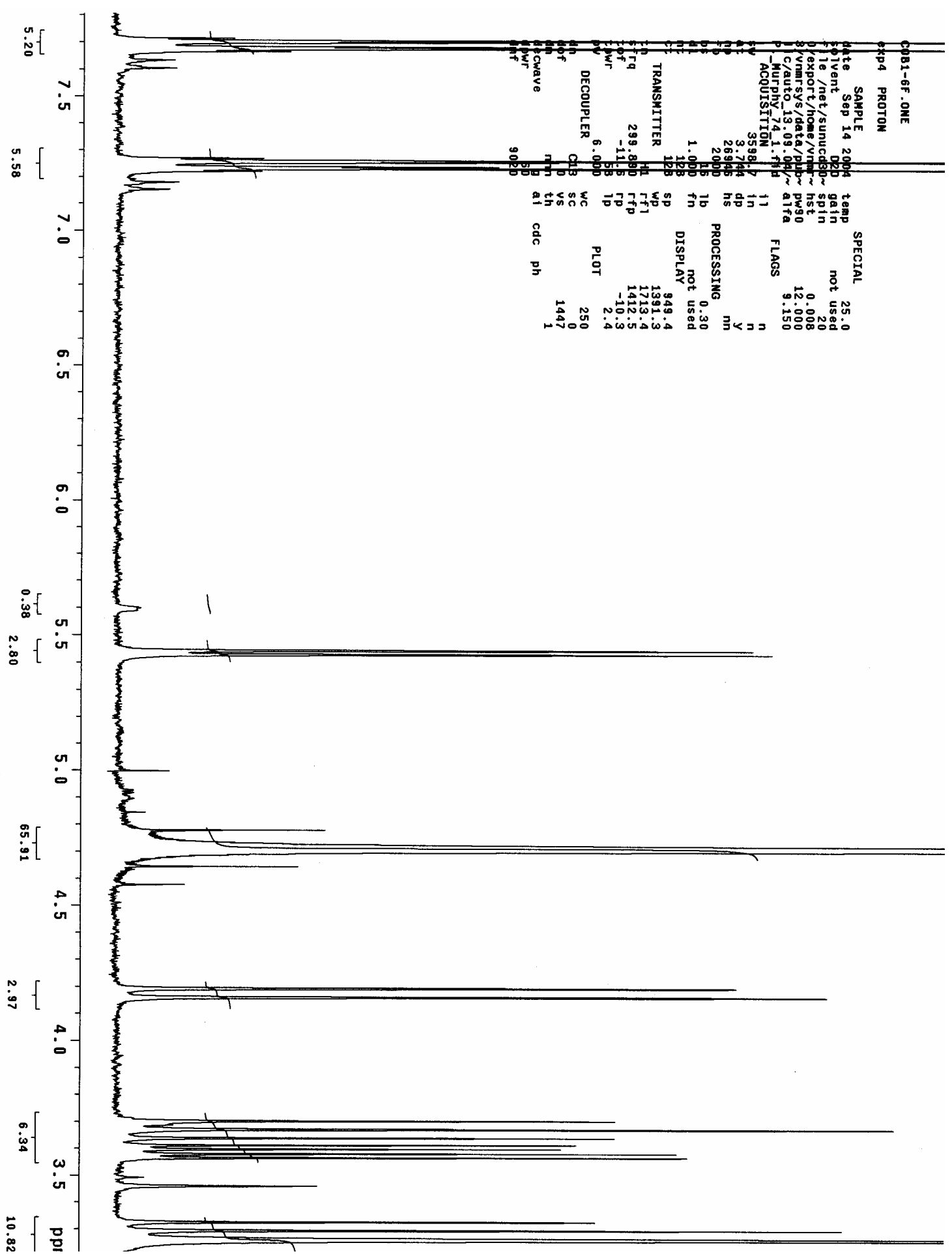


${ }^{1} \mathrm{H}-\mathrm{NMR}$<smiles>Cc1ccc(N(C)C(=O)CC(C(=O)O)C(O)OC(N)O)cc1</smiles>

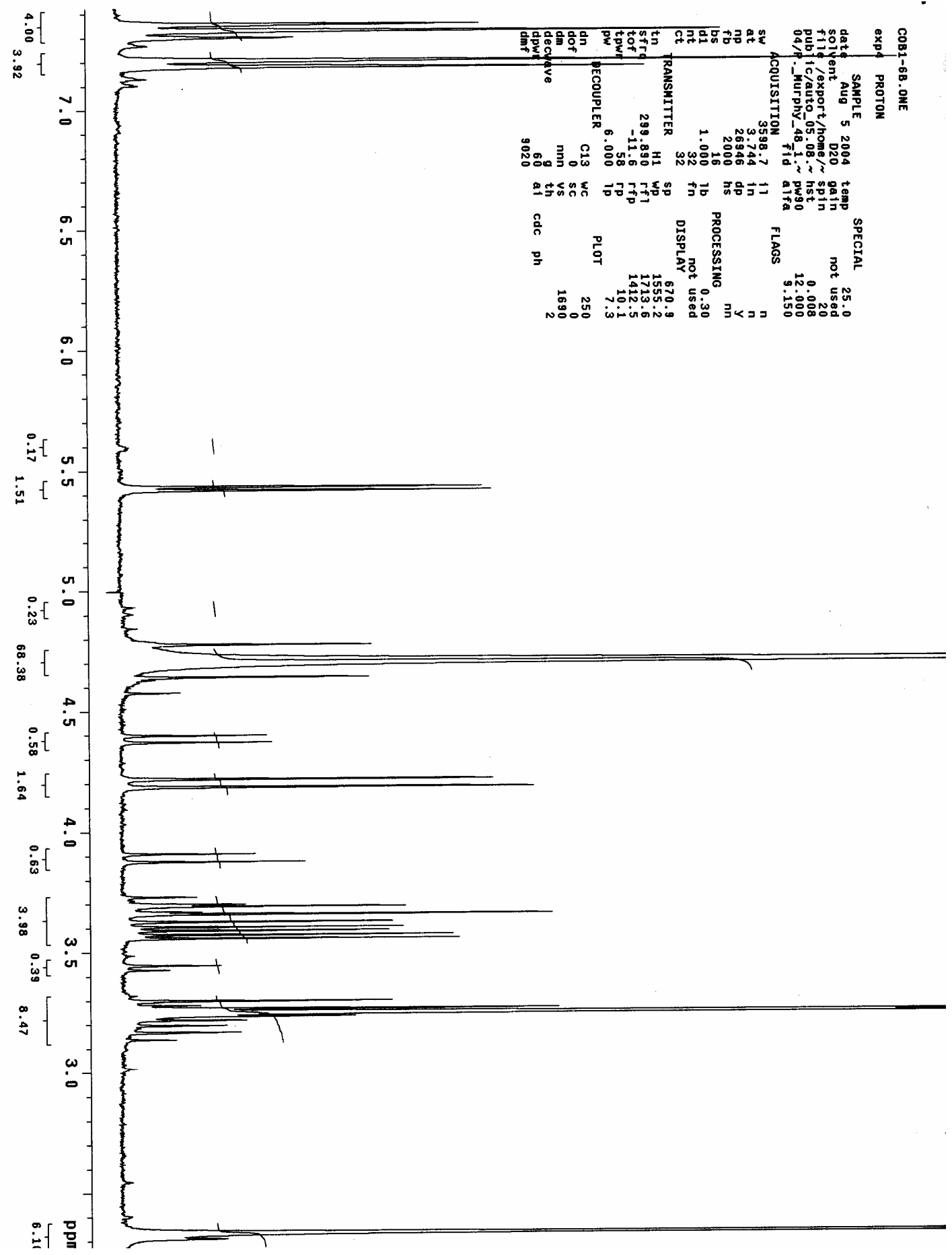


${ }^{1} \mathrm{H}-\mathrm{NMR}$<smiles>COc1ccc(N(C)C(=O)CC(C(=O)O)C(O)OC(N)C(=O)O)cc1</smiles>

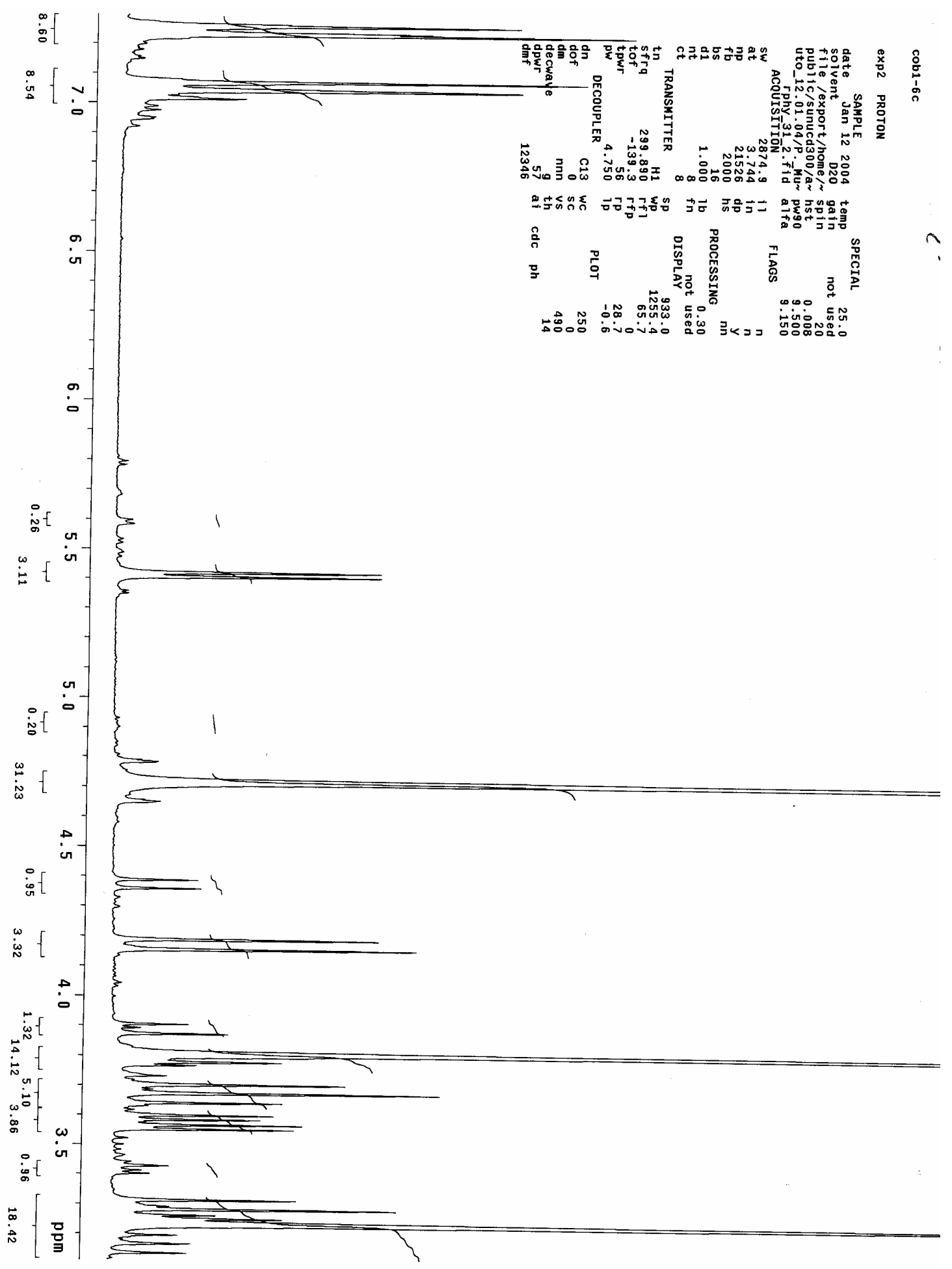


${ }^{1} \mathrm{H}-\mathrm{NMR}$<smiles>COC(=O)c1ccc(N(C)C(=O)CC(O)C(O)C(N)O)cc1</smiles>

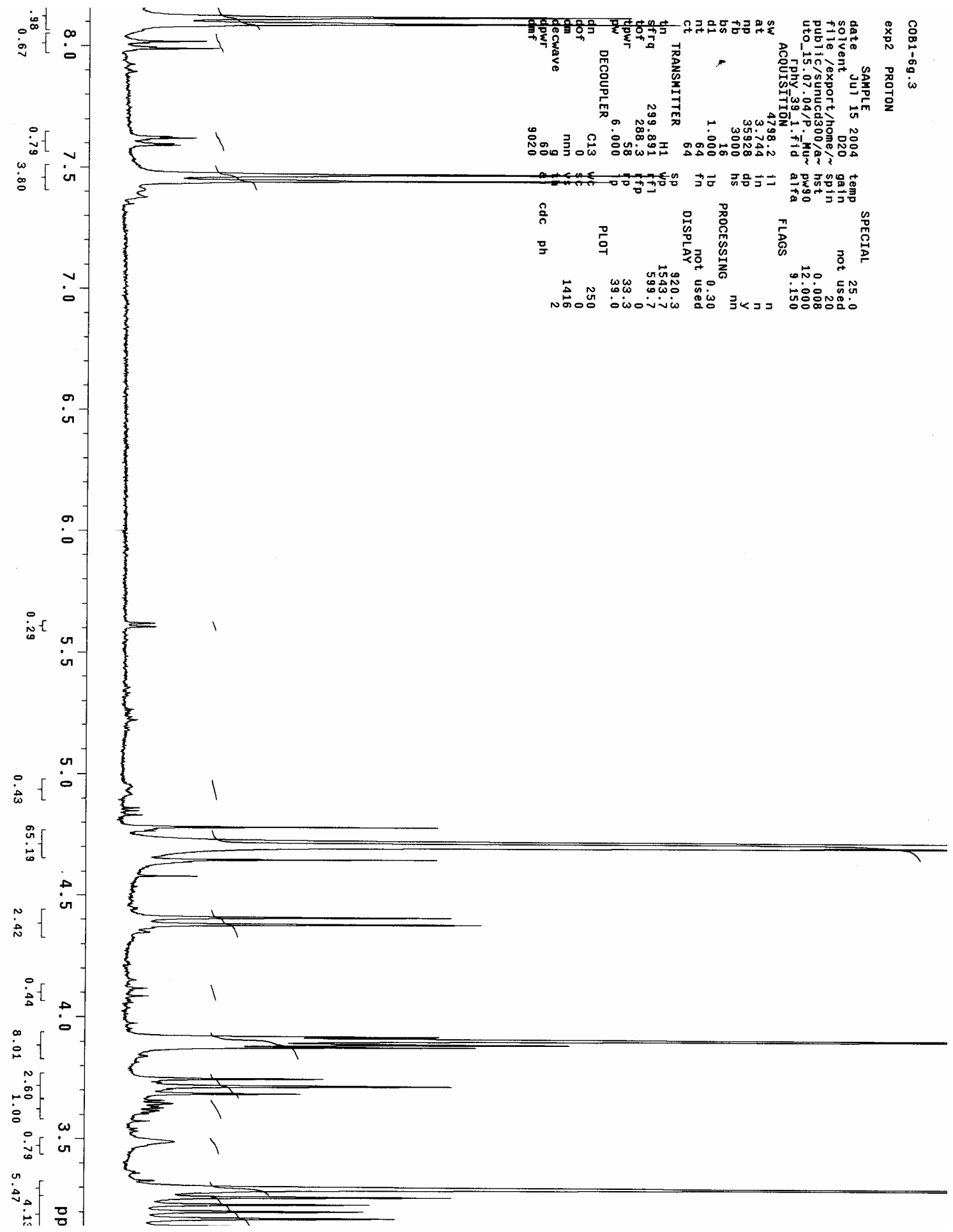


${ }^{1} \mathrm{H}-\mathrm{NMR}$

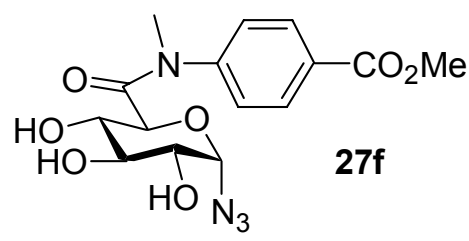

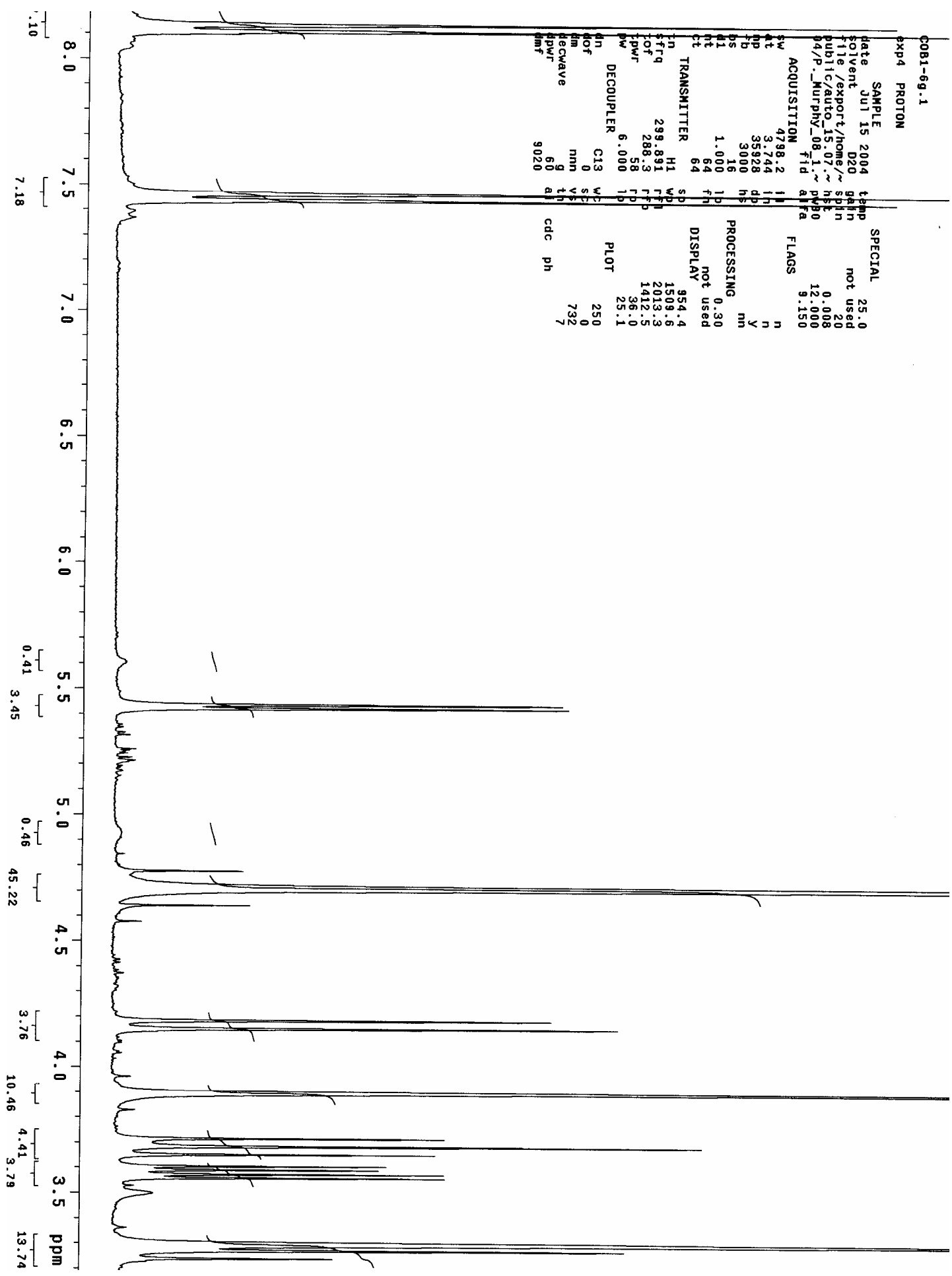


${ }^{1} \mathrm{H}-\mathrm{NMR}$<smiles>CN(C(=O)CC(O)C(O)O)c1ccccc1</smiles>

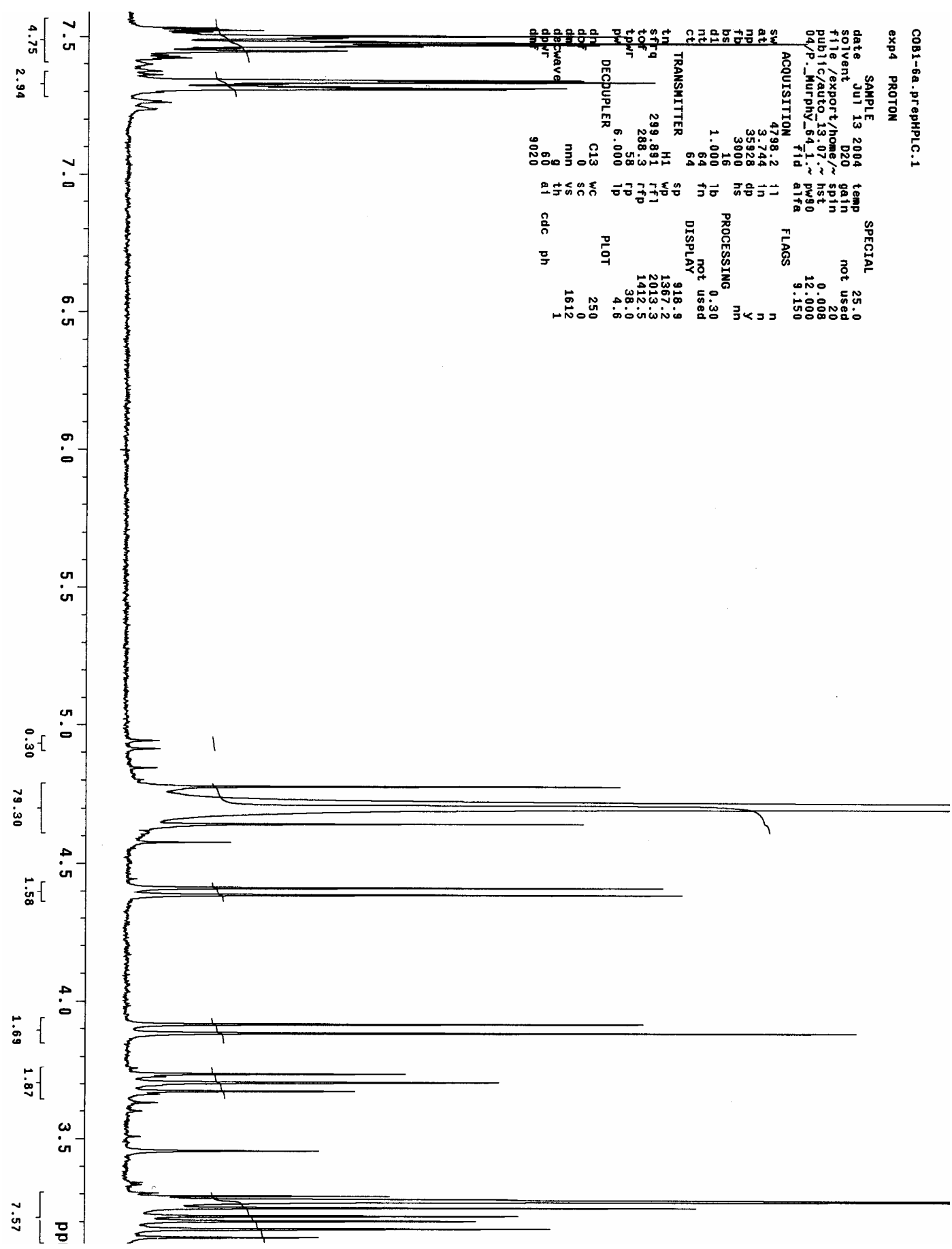


${ }^{1} \mathrm{H}-\mathrm{NMR}$<smiles>CN(C(=O)CC(O)C(O)CO)c1ccccc1</smiles>

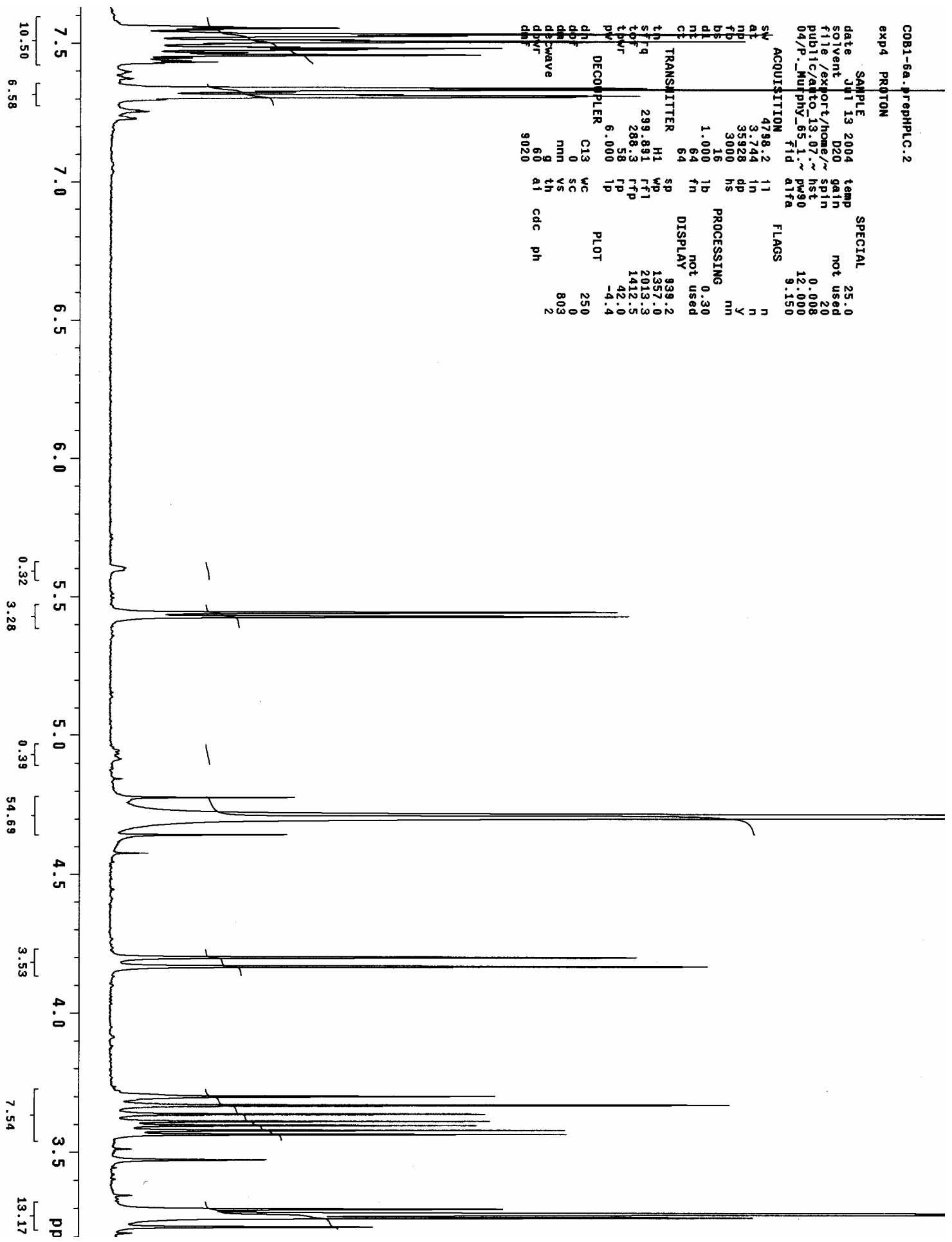




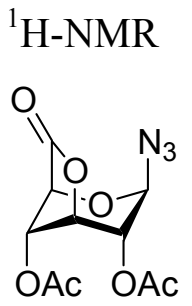

29

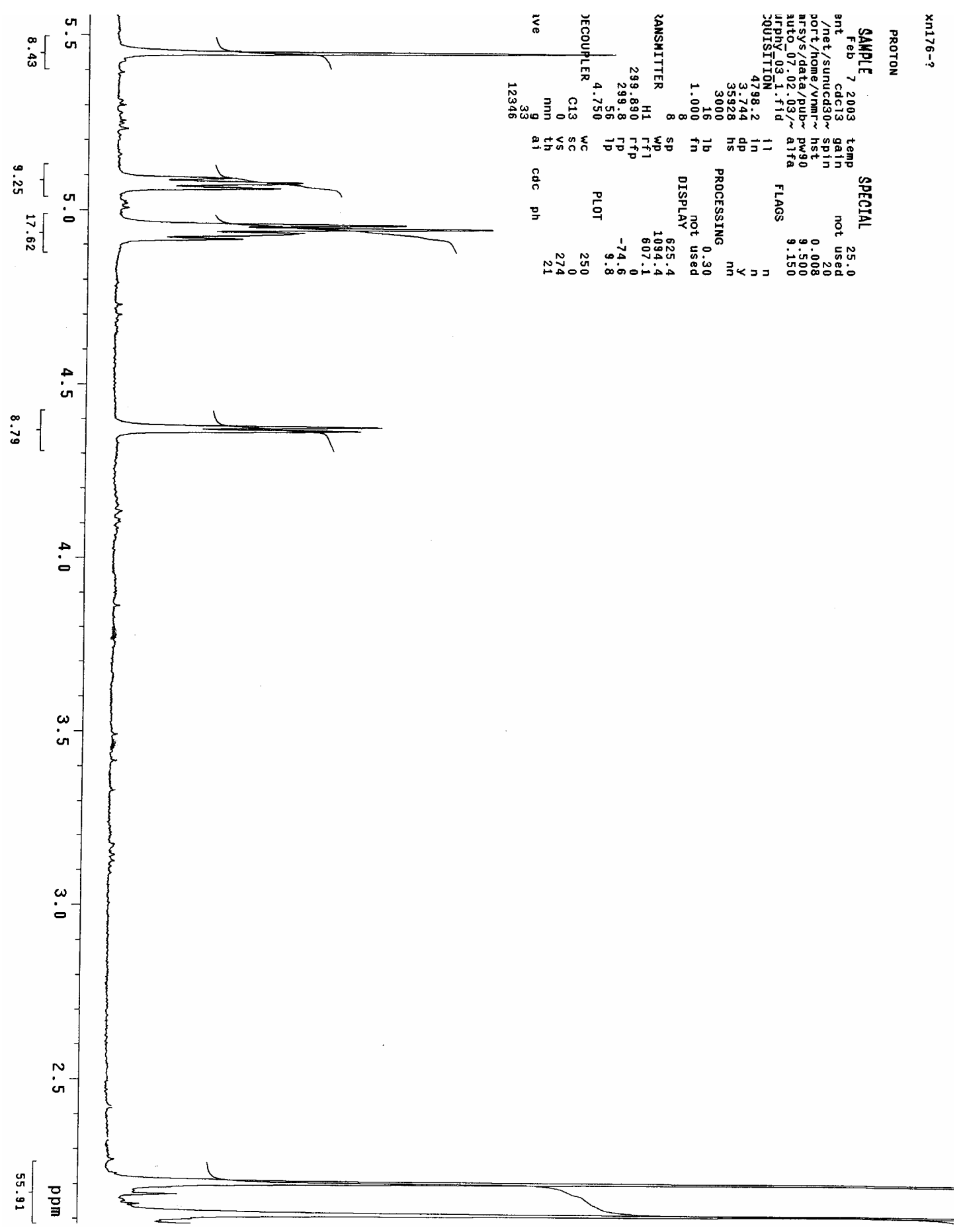




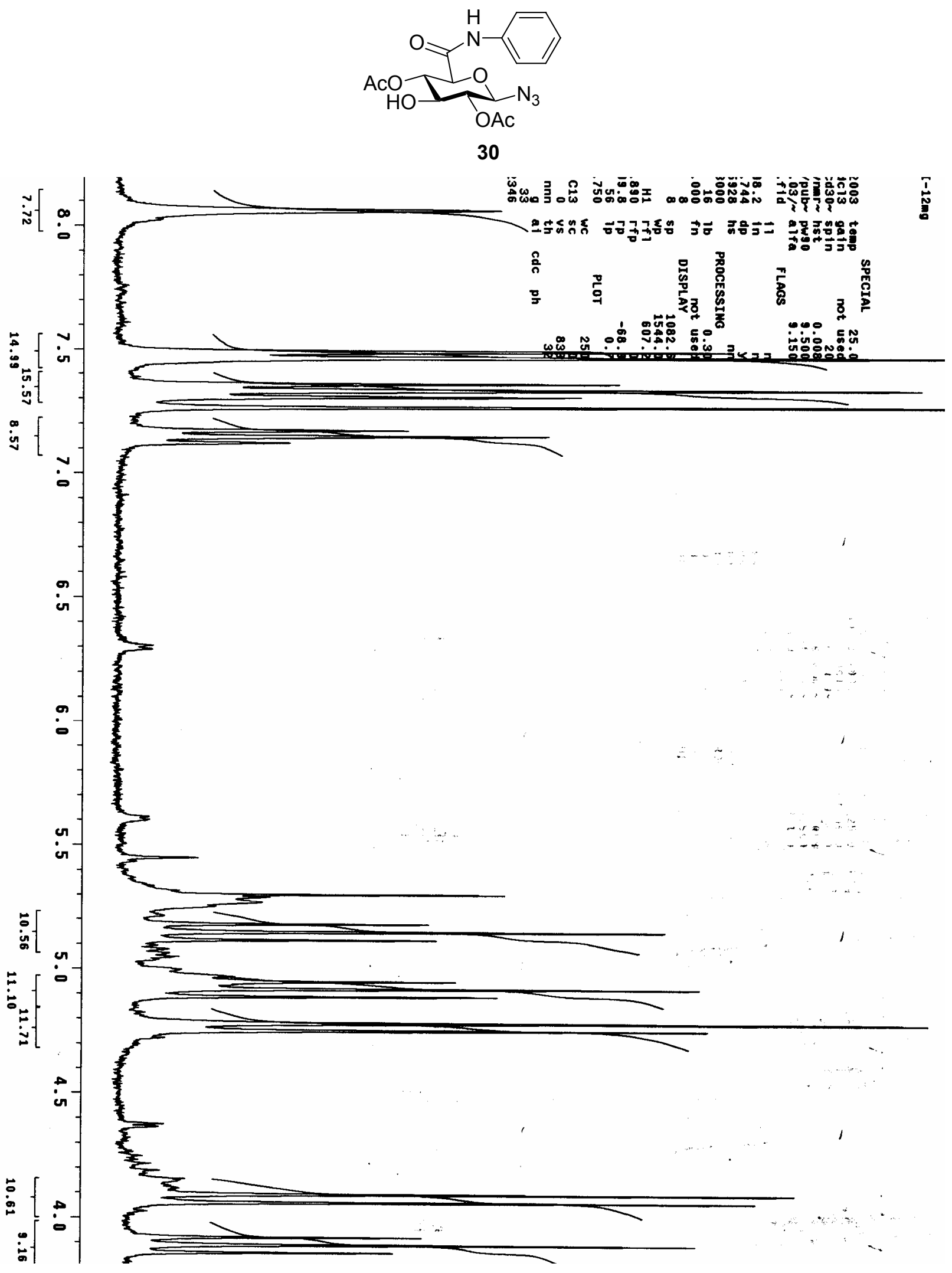




\section{D NOE 10}

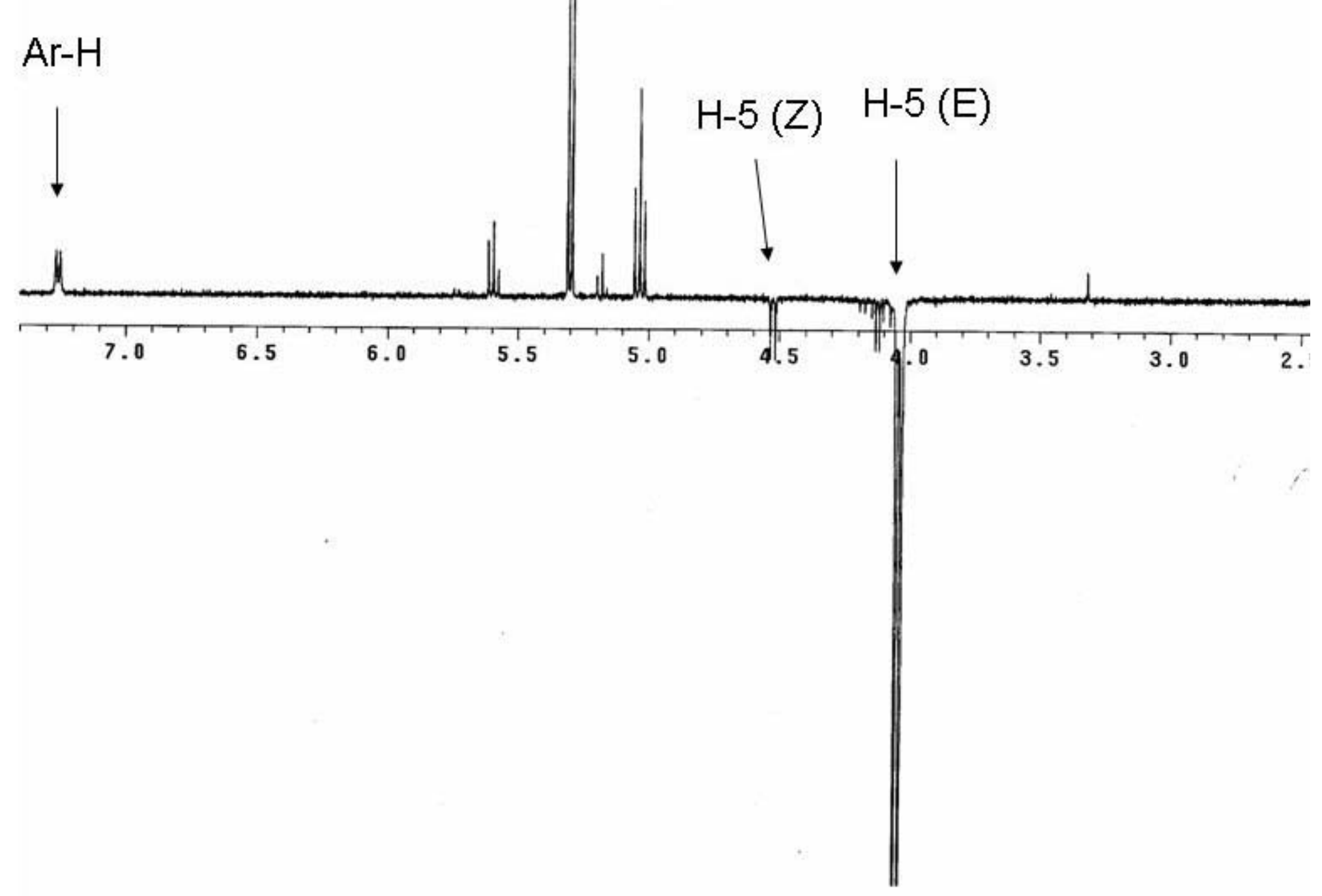




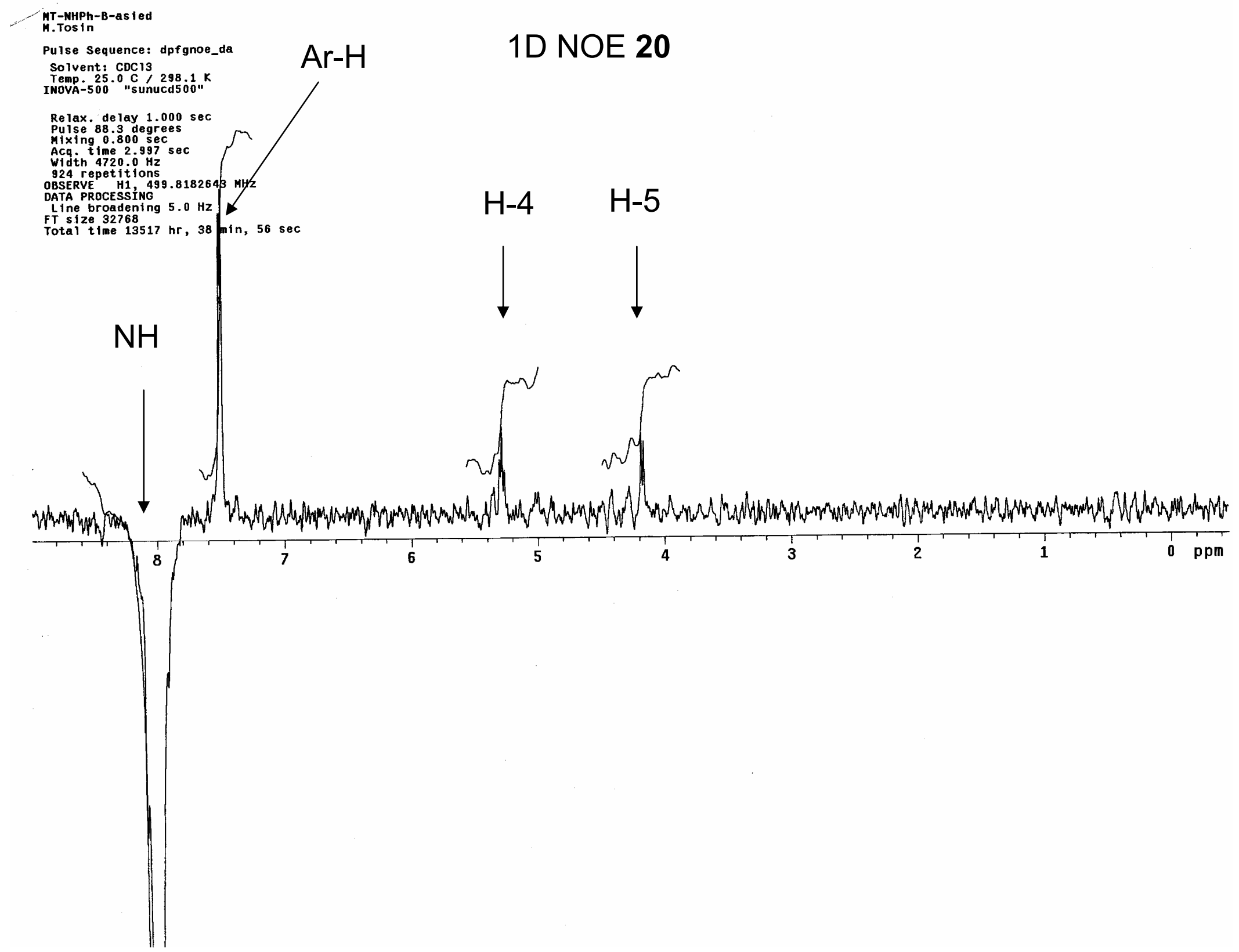



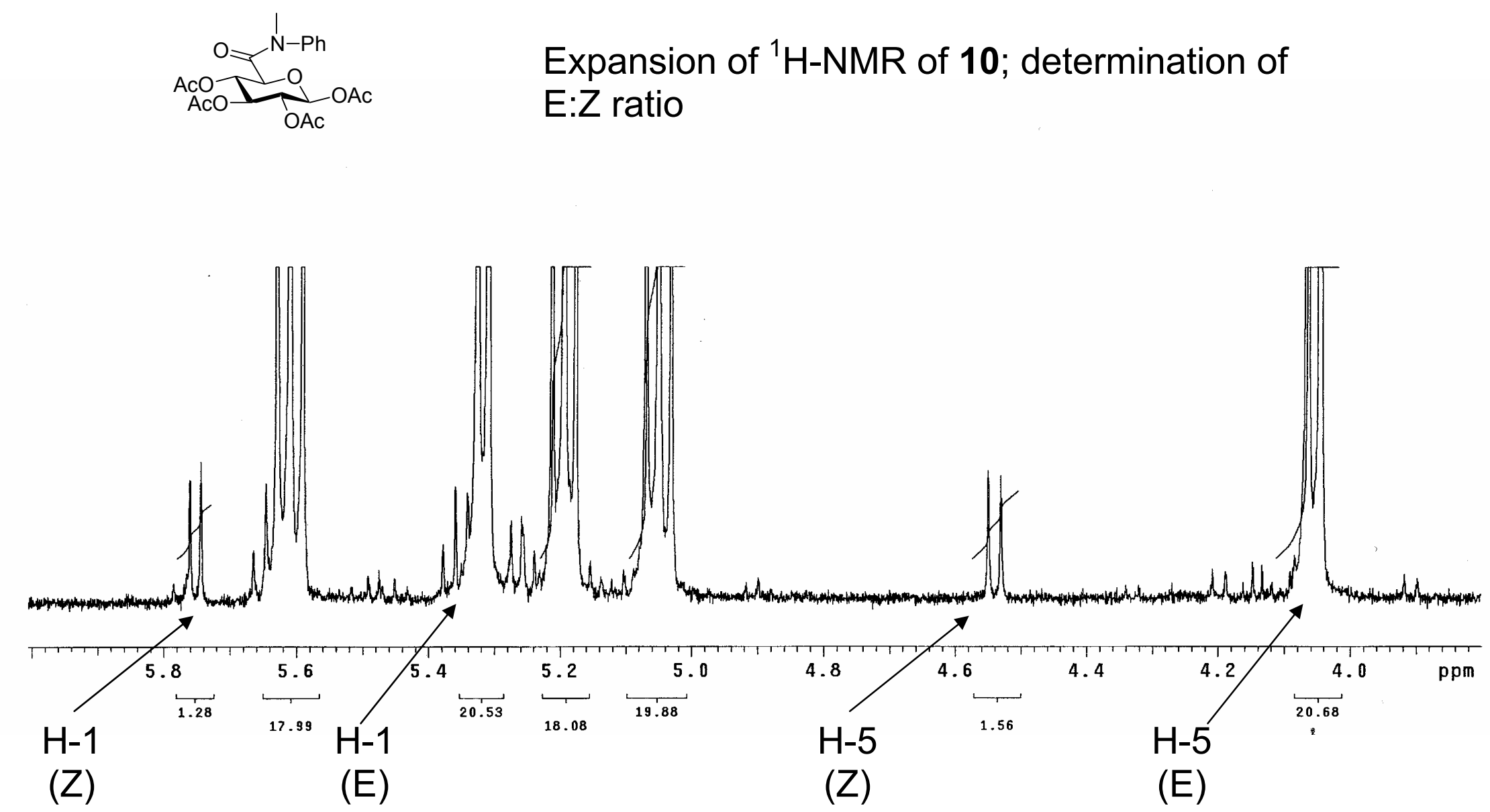